\title{
Tolerating Permanent and Transient Value Faults (Preliminary Version)
}

\author{
Zarko Milosevic \\ EPFL \\ Lausanne, Switzerland \\ zarko.milosevic@epfl.ch
}

\author{
Martin Hutle \\ Fraunhofer AISEC \\ Garching, Germany \\ martin.hutle@aisec.fraunhofer.de
}

\author{
André Schiper \\ EPFL \\ Lausanne, Switzerland \\ andre.schiper@epfl.ch
}

May 23, 2012

\begin{abstract}
Transmission faults allow us to reason about permanent and transient value faults in a uniform way. However, all existing solutions to consensus in this model are either in the synchronous system, or require strong conditions for termination, that exclude the case where all messages of a process can be corrupted. In this paper we introduce eventual consistency in order to overcome this limitation. Eventual consistency denotes the existence of rounds in which processes receive the same set of messages. We show how eventually consistent rounds can be simulated from eventually synchronous rounds, and how eventually consistent rounds can be used to solve consensus.

Depending on the nature and number of permanent and transient transmission faults, we obtain different conditions on $n$, the number of processes, in order to solve consensus in our weak model.
\end{abstract}

\section{Introduction}

Consensus is probably the most fundamental problem in fault-tolerant distributed computing. Consensus is related to the implementation of state machine replication, atomic broadcast, group membership, etc. The problem is defined over a set of processes $\Pi$, where each process $p \in \Pi$ has an initial value $v_{i}$, and requires that all processes agree on a common value.

Most research on consensus algorithms is considering component fault models, where faults are attached to a component that is either a process or a link. With respect to process/link faults, consensus can be considered with different fault assumptions. On the one end of the spectrum, processes/links can commit so called benign faults (processes fail only by crashing and links only loose mes- sages); on the other end, faulty processes/links can exhibit an arbitrary behavior. Furthermore, in the context of a component fault model, faults are mainly permanent: if a process or link commits a fault, the process/link is considered to be faulty during whole execution. It follows that not all components can be faulty, which is referred to as static faults. This explains that most research on consensus is about tolerating permanent and static process and/or link faults.

There are two major problems of a priori blaming some component for the failure [27, 28, 11]. First, it may lead to undesirable consequences: for example, in the classical Byzantine fault model, where a bounded number of processes can behave arbitrarily (even maliciously), the entire system will be considered faulty even if only one message from each process is received corrupted. Second, when solving consensus, faulty processes are typically not obliged to make a decision or they are allowed to decide differently than correct processes. Furthermore, when a process $q$ receives a corrupted message from $p$, it makes no difference for $q$ whether $p$ is faulty and therefore sends a message that was not consistent with the protocol, or the message is corrupted by the link between $p$ and $q$. Actually, for $q$ these two cases are indistinguishable.

These observations led to the definition of the transmission fault model that captures faults without blaming a specific component for the fault [27]. The transmission fault model is well-adapted to dynamic and transient faults. A transient fault is a non-permanent fault; a $d y$ namic fault is a fault that can affect any process/link in the system - as opposed to static faults that affect at most $f$ out of $n$ processes per run [5].

Consensus under transmission faults in a synchronous system has been considered initially in [27] and later in [30]. In [11], this work combined with ideas from [14], is extended to non-synchronous systems with only benign 
transmission faults, leading to the Heard-Of Model (HO model). The paper gives several consensus algorithms under benign transmission faults.

In [5], the HO model for benign faults is extended to value faults. There, consensus under transmission faults (both benign and value faults) is solved the first time in a non-synchronous setting. This was achieved by separating liveness conditions from safety conditions. For safety, only the number of corrupted messages is restricted, that is, in each round $r$ of the round based model, every process $p$ receives at most $\alpha$ corrupted messages ${ }^{1}$. However, for liveness, some additional assumptions are necessary, namely rounds in which some subset of processes does not receive any corrupted messages. ${ }^{2}$ This means that, despite the possibility to handle dynamic and transient value faults in a non-synchronous system, [5] cannot tolerate permanent faults located at a process $p$, where all messages from $p$ might be (always) corrupted.

This raises the following question: is it possible to design a consensus algorithm in the general transmission fault model, with non-synchronous assumptions, that does not require such a strong condition for liveness? If yes, such an algorithm would allow us to tolerate dynamic and transient faults, but also static and permanent faults. Furthermore, such an algorithm can be applied to a variety of system models: a partially synchronous system [12] with Byzantine processes, a partially synchronous system with Byzantine processes eventually restricted to "symmetrical faults" [31] (also termed "identical Byzantine" in [22]), a partially synchronous system with Byzantine processes, where, before stabilization time, in every round processes can receive some (bounded) number of corrupted messages from correct processes, etc. This spectrum of interpretations shows the benefit of considering the consensus problem in a model with (only) transmission faults.

Contribution. We give a positive answer to the above question by presenting several consensus algorithms for transmission faults (both benign and value faults) that do not require the strong conditions for liveness that exclude permanent faults. The key insight in achieving this goal is the introduction of the notion of eventual consistency, that turns out to be fundamental building block for solving consensus under transmission faults. Informally speaking, for round-based algorithms, eventual consistency denotes the existence of rounds in which processes receive the same set of messages.

We illustrate the relevance of eventual consistency by

\footnotetext{
${ }^{1}$ This assumption potentially allows corrupted messages on all links in a run; therefore it models dynamic faults.

${ }^{2}$ This assumption makes sense in the context of transient faults.
}

presenting three consensus algorithms for the transmission fault model that are inspired by well-known consensus algorithms $[12,10,20]$ defined for the classical Byzantine fault model [18]. All our algorithms require a round in which consistency eventually holds (processes receive the same set of messages). This round is used to bring the system in the univalent configuration, and later rounds are used to "detect" that the system entered a univalent configuration and allows processes to decide. Furthermore, we show how eventually consistent rounds can be simulated from eventually synchronous rounds in the presence of both static and dynamic value faults. Finally, we comment that eventual consistency can be achieved using authentication (e.g., digital signatures), a mechanism used for example in [20].

Organization of the paper. The rest of the paper is structured as follows. We describe the transmission fault model we consider in Section 2. The consensus problem is defined in Section 3, and in Section 4 we introduce the communication predicates that we consider in the paper, including eventual consistency. Section 5 shows how to simulate eventual consistency under weak communication predicates, while Section 6 shows how to solve consensus with eventual consistency. In Section 7 we discuss in detail the combination of one of the consensus algorithms and the eventual consistency simulation. As we show in Section 8, eventual consistency can be achieved also directly with authentication. In Section 9 we argue that Byzantine faults and permanent value faults located at a process are indistinguishable, and thus our algorithms also work (but not only) in a partial synchronous model with Byzantine processes. We refer to related work in Section 10 and conclude the paper in Section 11.

\section{Model}

We use a slightly extended version of the round-based model of [5]. In this model, we reason about faults only as transmission faults, without looking for a "culprit" for the fault [5]. Therefore there are no "faulty" processes and no state corruption in our model. ${ }^{3}$

Computations in this model are structured in rounds, which are communication-closed layers in the sense that any message sent in a round can be received only in that round. ${ }^{4}$ An algorithm $\mathcal{A}$ is specified by sending function $S_{p}^{r}$ and transition function $T_{p}^{r}$ for each round $r$ and

\footnotetext{
${ }^{3}$ Nevertheless, as explained in Section 9, the model can be used to reason about classical Byzantine faults.

${ }^{4}$ Note that the round structure of the model does not imply limits on the asynchrony of the system.
} 
process $p$. We now give a formal definition of the roundbased model considered, and introduce the notions of (i) the heard-of set $\mathrm{HO}(p, r)$, which captures synchrony and benign faults, (ii) the safe heard-of set $\operatorname{SHO}(p, r)$, which handles corruptions, i.e., captures communication safety properties, and (iii) consistency $\operatorname{CONS}(r)$, which is true in round $r$, if all processes receive the same set of messages at round $r$.

\subsection{Heard-Of Sets and Consistent Rounds}

Let $\Pi$ be a finite non-empty set of cardinality $n$, and let $\vec{M}$ be a set of messages (optionally including a null placeholder indicating the empty message). To each $p$ in $\Pi$, we associate a process, which consists of the following components: A set of states denoted by states $_{p}$, a subset init $_{p}$ of initial states, and for each positive integer $r$ called round number, a message-sending function $S_{p}^{r}$ mapping states $_{p}$ to a unique message from $\vec{M}$, and a state-transition function $T_{p}^{r}$ mapping states $_{p}$ and partial vectors (indexed by $\Pi$ ) of elements of $\vec{M}$ to states $_{p}$. The collection of processes is called an algorithm on $\Pi$. In each round $r$, a process $p$ : (1) applies $S_{p}^{r}$ to the current state and sends the message returned to each process, ${ }^{5}(2)$ determines the partial vector $\vec{\mu}_{p}^{r}$, formed by the messages that $p$ receives at round $r$, and (3) applies $T_{p}^{r}$ to its current state and $\vec{\mu}_{p}^{r}$. The partial vector $\vec{\mu}_{p}^{r}$ is called the reception vector of $p$ at round $r$.

Computation evolves in an infinite sequence of rounds. For each process $p$ and each round $r$, we introduce two subsets of $\Pi$. The first subset is the heard-of set, denoted $H O(p, r)$, which is the support of $\vec{\mu}_{p}^{r}$, i.e.,

$$
H O(p, r)=\left\{q \in \Pi: \vec{\mu}_{p}^{r}[q] \text { is defined }\right\} .
$$

The second subset is the safe heard-of set, denoted $S H O(p, r)$, and defined by

$$
\operatorname{SHO}(p, r)=\left\{q \in \Pi: \vec{\mu}_{p}^{r}[q]=S_{q}^{r}\left(s_{q}\right)\right\},
$$

where $s_{q}$ is $q$ 's state at the beginning of round $r$. In addition, for each round $r$, we define the consistency flag, denoted $\operatorname{CONS}(r)$, which is true if all processes receive the same set of messages in round $r$, i.e.,

$$
\operatorname{CONS}(r)=\left(\forall p, q \in \Pi^{2}: \vec{\mu}_{p}^{r}=\vec{\mu}_{q}^{r}\right) .
$$

From the sets $H O(p, r)$ and $\operatorname{SHO}(p, r)$, we form the altered heard-of set denoted $\mathrm{AHO}(p, r)$ as follows:

$$
A H O(p, r)=H O(p, r) \backslash S H O(p, r) .
$$

\footnotetext{
${ }^{5}$ W.l.o.g., the same message is sent to all.
}

For any round $r$, and for any set of rounds $\Phi$, we further define the safe kernel of $r$ resp. $\Phi$ :

$$
S K(r)=\bigcap_{p \in \Pi} S H O(p, r) \quad S K(\Phi)=\bigcap_{r \in \Phi} S K(r)
$$

The safe kernel consists of all processes whose messages were received correctly by all processes. We use also $S K=S K(\mathbb{N})$. Similarly, the altered span (of round $r$ ) denotes the set of processes from which at least one process received a corrupted message (at round $r$ ):

$$
A S(r)=\bigcup_{p \in \Pi} A H O(p, r) \quad A S=\bigcup_{r>0} A S(r)
$$

We also extend the notion of CONS in a natural way to a set $\Phi$ of rounds, i.e., $\operatorname{CONS}(\Phi)=\bigwedge_{r \in \Phi} \operatorname{CONS}(r)$.

\subsection{HO Machines}

A heard-of machine for a set of processes $\Pi$ is a pair $(\mathcal{A}, \mathcal{P})$, where $\mathcal{A}$ is an algorithm on $\Pi$, and $\mathcal{P}$ is a communication predicate, i.e., a predicate over the collection

$$
\left((H O(p, r), \operatorname{SHO}(p, r))_{p \in \Pi}, \operatorname{CONS}(r)\right)_{r>0}
$$

A run of an $\mathrm{HO}$ machine $M$ is entirely determined by the initial configuration (i.e., the collection of process initial states), and the collection of the reception vectors $\left(\vec{\mu}_{p}^{r}\right)_{p \in \Pi, r>0}$.

\subsection{Simulation of communication predi- cates}

In the paper we will need to simulate ${ }^{6}$ communication predicates $\mathcal{P}^{\prime}$ using some $\mathrm{HO}$ machine $M=(\mathcal{A}, \mathcal{P})$. Intuitively, in such a simulation, several rounds of $M$ will be used to simulate one round in which predicate $\mathcal{P}^{\prime}$ holds. If the run of $M$ consists of $k$ rounds, then algorithm $\mathcal{A}$ is a $k$ round simulation of $\mathcal{P}^{\prime}$ from $\mathcal{P}$.

Formally, let $k$ be any positive integer, and let $\mathcal{A}$ be an algorithm that maintains a variable $m_{p} \in \vec{M}$ and $M s g_{p} \in$ $\vec{M}^{n}$ at every process $p$. We call macro-round $\rho$ the sequence of the $k$ consecutive round $k(\rho-1)+1, \ldots, k \rho$. The variable $m_{p}$ is an input variable that can be set externally in every macro-round. ${ }^{7}$ The value of $m_{p}$ at the beginning

\footnotetext{
${ }^{6}$ The notion of a simulation differs from the notion of a translation of the HO model for benign faults. A translation establishes a relation purely based on connectivity, while with value faults, also some computation is involved. Because of this, we decided thus to use the term simulation instead.

${ }^{7}$ The sending function in a simulation algorithm is thus a function that maps states $_{p}$ and the input from $\vec{M}$ to a unique message from $\vec{M}$; while the state-transition function $T_{p}^{r}$ is a function that maps states $s_{p}$, the input from $\vec{M}$, and a partial vector (indexed by $\Pi$ ) of elements of $\vec{M}$ to states $_{p}$.
} 
of macro-round $\rho$ is denoted $m_{p}^{(\rho)}$, and the value of $M s g_{p}$ at the end of macro-round $\rho$ is denoted $M s g_{p}^{(\rho)}$. For the macro-round $\rho$, we define in analogy to the definitions of Section 2.1:

$$
\begin{aligned}
H O(p, \rho) & =\left\{q \in \Pi: \operatorname{Msg}_{p}^{(\rho)}[q] \text { is defined }\right\} \\
\operatorname{SHO}(p, \rho) & =\left\{q \in \Pi: \operatorname{Msg}_{p}^{(\rho)}[q]=m_{q}^{(\rho)}\right\} \\
\operatorname{CONS}(\rho) & =\left(\forall p, q \in \Pi^{2}: M s g_{p}^{(\rho)}=M s g_{q}^{(\rho)}\right)
\end{aligned}
$$

We say that the $\mathrm{HO}$ machine $M=(\mathcal{A}, \mathcal{P})$ simulates the communication predicate $\mathcal{P}^{\prime}$ in $k$ rounds if for

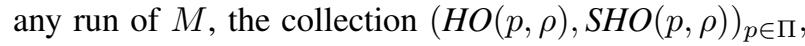
$\operatorname{CONS}(\rho))_{\rho>0}$ satisfies predicate $\mathcal{P}^{\prime}$.

Given a simulation $\mathcal{A}$ of $\mathcal{P}^{\prime}$ from $\mathcal{P}$, any problem that can be solved with $\mathcal{P}^{\prime}$ by algorithm $\mathcal{A}^{\prime}$ can be solved with $\mathcal{P}$ instead by simply simulating rounds of the algorithm $\mathcal{A}^{\prime}$ using algorithm $\mathcal{A}$. In such a composed algorithm, the input variable $m_{p}^{(\rho)}$ of algorithm $\mathcal{A}$ is set at each macroround $\rho$ to the value returned by the sending function of $\mathcal{A}^{\prime}$, and the transition function of $\mathcal{A}^{\prime}$ is applied to the output $M s g_{p}^{(\rho)}$ of algorithm $\mathcal{A}$.

\section{Consensus}

Let $V$ be (non-empty) totally ordered set. In the consensus problem every process $p$ has an initial value init $_{p} \in V$ and decides irrevocably on a decision value, fulfilling:

Integrity: If all processes have the same initial value this is the only possible decision value.

Agreement: No two processes may decide differently.

Termination: All processes eventually decide.

Since, contrary to classical approaches, there is no deviation according to $T_{p}^{r}$, and thus we do not have the notion of a faulty process, the upper specification makes no exemption: all processes must decide the initial value in the Integrity clause, and all processes must make a decision by the Termination clause.

Formally, an $\mathrm{HO}$ machine $(\mathcal{A}, \mathcal{P})$ solves consensus, if any run for which $\mathcal{P}$ holds, satisfies Integrity, Agreement, and Termination. To make this definition non-trivial, we assume that the set of $\mathrm{HO}$ and $\mathrm{SHO}$ collections for which $\mathcal{P}$ holds is non-empty.

\section{Communication predicates}

In this section we introduce the communication predicates that will be used in the paper. As already mentioned, we reason about faults only as transmission faults. This allows us to deal with both permanent and transient faults, but also with static and dynamic faults.

\subsection{Predicates that capture static and dy- namic value faults}

A dynamic fault is a fault that can affect any link in the system - as opposed to static faults that affect the links of at most $f$ out of $n$ processes per run [5]. We start with static faults:

$$
\mathcal{P}_{\text {stat }}^{f}::|A S| \leq f
$$

with $f \in N$ and $N=\{0, \ldots, n\} . \mathcal{P}_{\text {stat }}$ is the name of the predicate, and $f$ is a free parameter. $\mathcal{P}_{\text {stat }}^{f}$ is a safety predicate that models static faults, where corrupted messages are received only from a set of $f$ processes. In Section 9 we will argue that such an assumption corresponds to a system with at most $f$ Byzantine processes.

For our algorithms we will also consider the weaker safety predicate $\mathcal{P}_{\text {dyn }}^{f}\left(\forall f \in N, \mathcal{P}_{\text {stat }}^{f}\right.$ implies $\left.\mathcal{P}_{\text {dyn }}^{f}\right)$ that restricts the number of corrupted messages only per round and per process:

$$
\mathcal{P}_{d y n}^{f}:: \forall r>0, \forall p \in \Pi:|A H O(p, r)| \leq f
$$

with $f \in N$ and $0 \leq f \leq n$. Predicate $\mathcal{P}_{d y n}^{f}$ potentially allows corrupted messages on all links in a run, it therefore models dynamic value faults.

\subsection{Predicates that restrict asynchrony of communi- cation and dynamism of faults}

Predicates $\mathcal{P}_{\text {stat }}$ and $\mathcal{P}_{\text {dyn }}$ only restrict the number of value faults; however, it does not tell us anything about liveness of communication. From [13] we know that we cannot solve consensus in an asynchronous system if all messages sent by one process may be lost. On the other hand, Santoro and Widmayer [27] showed that consensus is impossible to solve in a synchronous system if, at each time unit, there is one process whose messages may be lost. Therefore, in order to solve consensus we need to restrict asynchrony of communication and dynamism of faults.

A synchronous system could be modeled as follows:

$$
\mathcal{P}_{S K}^{f}::|S K| \geq n-f
$$


$\mathcal{P}_{S K}^{f}$ requires that there is a set of processes (safe kernel) of size $n-f$ whose messages are correctly received in every round. From

$$
\forall f \in N, \mathcal{P}_{S K}^{f} \Rightarrow \mathcal{P}_{\text {stat }}^{f}
$$

it follows that $\mathcal{P}_{S K}^{f}$ implies static faults only. However, we want to study consensus with dynamic faults. We consider therefore the following predicate:

$$
\begin{aligned}
\mathcal{P}_{\diamond S K}^{f, k}: & : \forall r>0 \exists r_{o}>r, \Phi=\left\{r_{0}, \ldots, r_{0}+k-1\right\}: \\
& |S K(\Phi)| \geq n-f
\end{aligned}
$$

with $f \in N$ and $k>0$. This predicate (repeatedly) requires a safe kernel of size $n-f$ only eventually and only for $k$ rounds. It also restrict the dynamism of value faults during these $k$ round; i.e., corrupted messages can only be received from at most $f$ processes.

In the paper we will consider $\mathcal{P}_{\diamond S K}$ always in conjunction, either with $\mathcal{P}_{\text {stat }}$ or $\mathcal{P}_{d y n}$. When we assume $\mathcal{P}_{\diamond S K}$ with $\mathcal{P}_{\text {stat }}$, i.e., $\mathcal{P}_{\diamond S K}^{f, k} \wedge \mathcal{P}_{\text {stat }}^{f}$, transmission value faults are static (benign transmission faults are not restricted, so they can be dynamic). On the other hand, when we assume $\mathcal{P}_{\diamond S K}$ with $\mathcal{P}_{d y n}$, i.e., $\mathcal{P}_{\diamond S K}^{f, k} \wedge \mathcal{P}_{d y n}^{\alpha}$ with $f \leq \alpha$, transmission value faults are no more static: $\mathcal{P}_{\diamond S K}^{f, k}$ alone does not imply $\mathcal{P}_{\text {stat }}^{f^{\prime}}$ for any $f^{\prime}<n$.

\subsection{Permanent versus Transient Faults}

Both predicates, $\mathcal{P}_{\text {stat }} \wedge \mathcal{P}_{\diamond S K}$ and $\mathcal{P}_{\text {dyn }} \wedge \mathcal{P}_{\diamond S K}$ allow permanent faults. Consider for example a run and a process $p$, where every process receives a corrupted message from $p$ in every round:

$$
\forall q \in \Pi, r>0: p \notin S H O(q, r)
$$

and all other messages are received correctly. Such a run is included in the set of runs given by $\mathcal{P}_{\text {stat }} \wedge \mathcal{P}_{\diamond S K}$ and $\mathcal{P}_{\text {dyn }} \wedge \mathcal{P}_{\diamond S K}$, and thus our algorithms can solve consensus in such a run. More precisely, $\mathcal{P}_{\text {stat }}^{f} \wedge \mathcal{P}_{\diamond S K}^{f}$ and $\mathcal{P}_{d y n}^{f} \wedge \mathcal{P}_{\diamond S K}^{f}$ permits the existence of up to $f$ such processes. As pointed out in Section 9, this allows our algorithms to solve consensus also, e.g., in classical models with Byzantine faults. This contrasts with previous work [5], where, although also $\mathcal{P}_{d y n}$ is considered (named $\mathcal{P}_{\alpha}$ there), eventually there has to be a round, where a sufficiently large subset of processes do not receive any corrupted messages. There, (most) faults have to be transient.

\subsection{Eventual Consistency}

In this section we introduce the notion of eventual consistency that turns out to be a fundamental building block for solving consensus under transmission value faults. Eventual consistency abstracts the major complexity present when solving consensus under the weak communication predicates presented above. Therefore eventual consistency allows us to express consensus algorithms in a very concise and elegant way.

Informally speaking, eventual consistency combines the requirement of a consistent round $(\operatorname{CONS}(r)$ in our model) with some requirements on liveness and safety of communication. It can be seen as an eventual version of interactive consistency [23]. An algorithm that solves interactive consistency allows correct processes to come to a consistent view of the initial values of the processes, i.e., all processes agree on a vector, where $n-f$ entries correspond to the initial values of the respective process ( $f$ is the number of faulty processes in a model with process faults, and just a parameter in our case).

Interactive consistency, when seen as a communication primitive, can be captured by the following predicate:

$$
\mathcal{P}_{I C}^{f}::|S K| \geq n-f \wedge \forall r>0: \operatorname{CONS}(r)
$$

When we express the result of [23] in our model, their algorithm allows a $f+1$ round simulation of $\mathcal{P}_{I C}^{f}$ from $\mathcal{P}_{S K}^{f}$ if $n>3 f$. Note that $\forall f \in N, \mathcal{P}_{I C}^{f} \Rightarrow \mathcal{P}_{\text {stat }}^{f}$.

Instead of $\mathcal{P}_{I C}$, we introduce a weaker predicate. We call the predicate eventual consistency and define it as follows:

$$
\mathcal{P}_{\diamond \text { cons }}^{f}:: \forall r>0 \exists r_{o}>r:\left|S K\left(r_{0}\right)\right| \geq n-f \wedge \operatorname{CONS}\left(r_{0}\right)
$$

This predicate requires that there is always eventually a consistent round with a safe kernel of size $n-f$. In contrast to $\mathcal{P}_{S K}^{f}$ and $\mathcal{P}_{I C}$, this predicate requires these safe kernels only eventually and then only for a single round. Also faults are no more static: $\mathcal{P}_{\diamond \text { cons }}^{f}$ alone does not imply $\mathcal{P}_{\text {stat }}^{f^{\prime}}$ for any $f^{\prime}<n$. Note that $\mathcal{P}_{\diamond \text { cons }}^{f}$ is a stronger predicate than $\mathcal{P}_{\diamond S K}^{f, 1}$ : although both predicates require a safe kernel of size $n-f$ and both restrict the dynamism of value faults for a single round, $\mathcal{P}_{\diamond \text { cons }}^{f}$ in addition requires that consistency holds during this round, i.e., for any two processes $p$ and $q$ we have $\vec{\mu}_{p}=\vec{\mu}_{q}$.

However, $\mathcal{P}_{\diamond \text { cons }}$ can be simulated from $\mathcal{P}_{\diamond S K}$. In the next section, we give two such simulations, and then establish the link to solving consensus. 


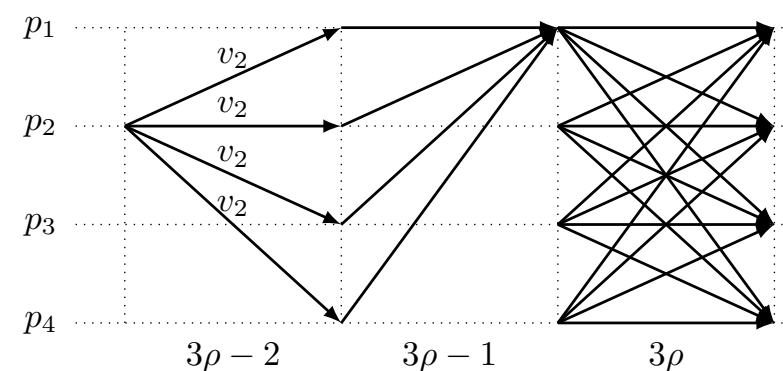

Figure 1: Algorithm 1 from the point of view of $v_{2}$ sent by $p_{2}\left(p_{1}\right.$ is the coordinator, $n=4, f=1$ ).

\section{Simulating eventual consistency $\mathcal{P}_{\diamond c o n s}$ from eventually safe kernels $\mathcal{P}_{\diamond S K}$}

In this section we give two simulations of $\mathcal{P}_{\diamond \text { cons }}$ from $\mathcal{P}_{\Delta S K}$, one in the presence of only static value faults $\left(\mathcal{P}_{\text {stat }}\right)$, and the other in the presence of dynamic (and static) value faults $\left(\mathcal{P}_{d y n}\right)$. As we show, the first simulation requires a smaller number of processes in order to tolerate a given number of transmission value faults. Then we introduce a generic predicate $\mathcal{P}_{\Delta c o n s \oplus S K}$ that can be simulated from $\mathcal{P}_{\diamond S K}$. The predicate $\mathcal{P}_{\diamond \text { cons } \oplus S K}$, in conjunction with $\mathcal{P}_{\text {dyn }}$ or $\mathcal{P}_{\text {stat }}$, is later used in Section 6 to solve consensus.

\subsection{Simulation in the presence of only static value faults}

Algorithm 1 is a 3-round simulation of $\mathcal{P}_{\diamond \text { cons }}^{f} \wedge \mathcal{P}_{\text {stat }}^{f}$ from $\mathcal{P}_{\diamond S K}^{3(f+1)} \wedge \mathcal{P}_{\text {stat }}^{f}$ inspired by [10]. It ensures consistency during a sequence of rounds where the size of the kernel is at least $n-f$ (the corrupted messages can be received only from at most $f$ processes). Moreover, it preserves $\mathcal{P}_{\text {stat }}^{f}$, i.e., if $\mathcal{P}_{\text {stat }}^{f}$ holds for basic rounds, then $\mathcal{P}_{\text {stat }}^{f}$ holds also for the macro-rounds obtained by the 3round simulation using Algorithm 1. It requires $n>3 f$. As already mentioned in Section 2.3, a simulation is an algorithm that maintains at each process $p$ two variables: an input variable $m_{p}$ that is set at the beginning of every macro-round $\rho$ (line 7), and an output variable $M s g_{p}$ whose value is considered at the end of every macro-round $\rho$ (lines 24 and 26). The special value $\perp$ represents the case when a (reception) vector does not contain a message from the respective process.

Algorithm 1 is a coordinator-based algorithm, where

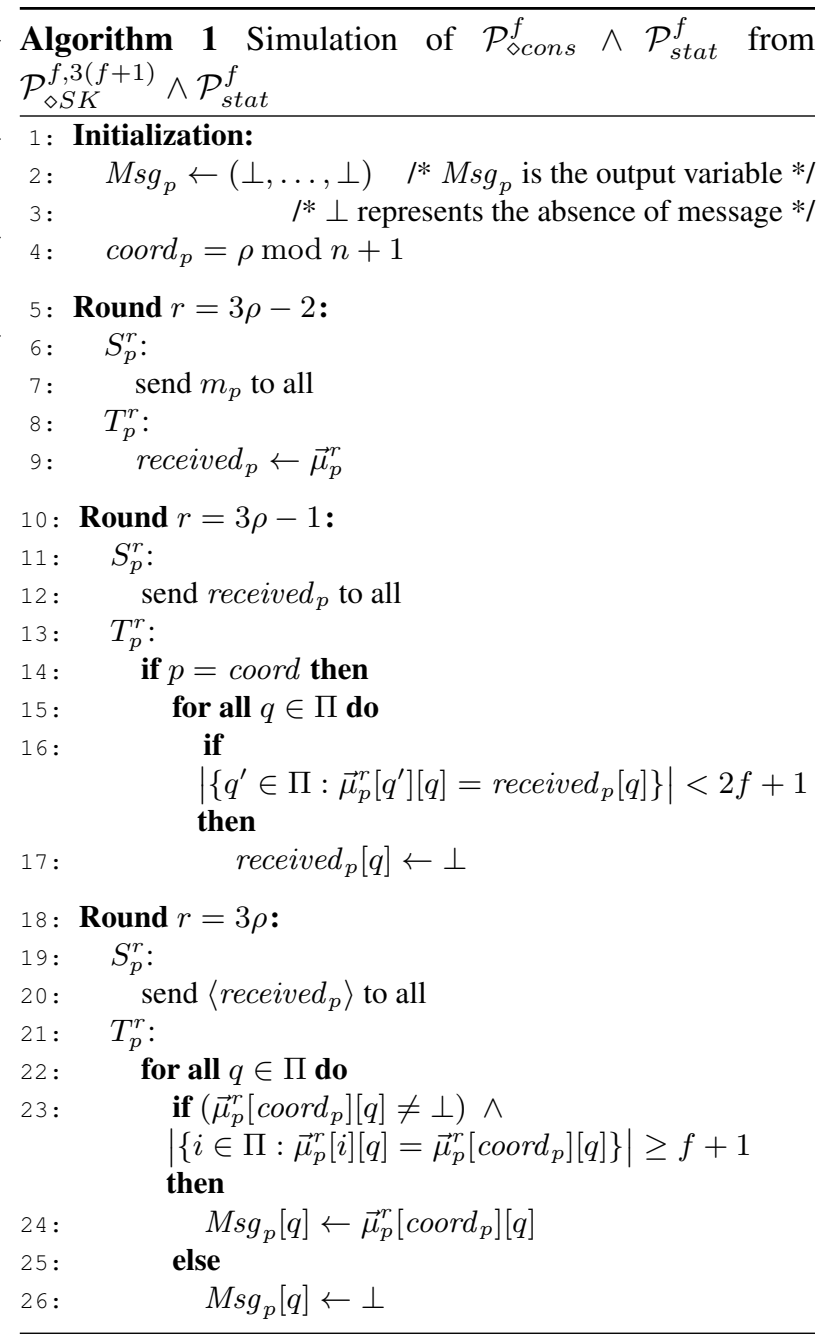

the coordinator is chosen using a rotating coordinator strategy: the coordinator of macro-round $\rho$ is process $\rho \bmod n+1$; in Algorithm 1 the variable coord refers to this process. We describe Algorithm 1 from the point of view of the message $v_{2}$ that is sent by process $p_{2}$ using Figure 1. Assume that process $p_{1}$ is the coordinator. In round $3 \rho-2$, process $p_{2}$ sends the message $v_{2}$ to all. In rounds $3 \rho-1$ and $3 \rho$ of Algorithm 1, the processes send messages that contain a vector of those messages received in round $3 \rho-2$. In this description we focus only on those elements of the vectors that are related to message $v_{2}$ that is sent by process $p_{2}$ in macro-round $\rho$. In round $3 \rho-1$, all processes send the value received from $p_{2}$ to all. ${ }^{8}$ The coordinator then compares the value received from $p_{2}$, say $v_{2}$, in round $3 \rho-2$ with the value indirectly received from

\footnotetext{
${ }^{8}$ At line 16 , the reception vector $\vec{\mu}_{p}^{r}$ is a vector of vectors: $\vec{\mu}_{p}^{r}\left[q^{\prime}\right]$ is the vector $p$ has received from $q^{\prime}$, and $\vec{\mu}_{p}^{r}\left[q^{\prime}\right][q]$ is element $q$ of this vector.
} 
the other processes.

If at least $2 f+1$ values $v_{2}$ have been received by the coordinator $p_{1}$, then $p_{1}$ keeps $v_{2}$ as the message received from $p_{2}$. Otherwise $p_{1}$ sets the message received from $p_{2}$ to $\perp$ (line 17). This guarantees that if $p_{1}$ keeps $v_{2}$, then at least $f+1$ processes have received $v_{2}$ from $p_{2}$ in round $3 \rho-2$. Finally, in round $3 \rho$ every process sends the value received from $p_{2}$ in round $3 \rho-2$ to all. The final value adopted as message received from $p_{2}$ at the end of round $3 \rho$ (and therefore at the end of macro-round $\rho$ ) is computed as follows at each process $p_{i}$. Let $v a l_{i}$ be the value received from coordinator $p_{1}$ in round $3 \rho$. If $v a l_{i}=\perp$ then $p_{i}$ receives $\perp$ from $p_{2}$. Process $p_{i}$ receives $\perp$ from $p_{2}$ in another case: if $p_{i}$ did not receive $f+1$ values equal to $\mathrm{val}_{i}$ in round $3 \rho$. Otherwise, at least $f+1$ values received by $p_{i}$ in round $3 \rho$ are equal to $v a l_{i}$, and $p_{i}$ adopts $v a l_{i}$ as message received from $p_{2}$ in macro-round $\rho$.

Algorithm 1 relies on a coordinator for ensuring $\mathcal{P}_{\diamond c o n s}^{f}$ : all processes assign to $M s g_{p}$ the value received from the coordinator in round $3 \rho$ (see line 24). This is achieved during a macro-round in which the size of the safe kernel is at least $n-f$, with the coordinator in the safe kernel. The rotating coordinator strategy ensures the existence of such a macro-round. Consider Figure 2 that illustrates the mechanism for ensuring consistency from the point of view of the message sent by process $p_{4}$ and received by process $p_{3}$. The coordinator adopts value $v_{1}$ as the message sent by process $p_{4}$ in round $3 \rho-1$ (line 16) since it is forwarded by $2 f+1$ processes. This ensures that the value $v_{1}$ sent by the coordinator in round $3 \rho$ is also sent by at least $f$ more processes from the safe kernel in round $3 \rho$. Therefore, the value sent by the coordinator satisfies the condition of line 23 at all processes and is therefore assigned to $\mathrm{Msg}_{p}\left[p_{4}\right]$ by all processes at line 24 .

Using Figure 3, we now explain how Algorithm 1 preserves $\mathcal{P}_{\text {stat }}^{f}$. Figure 3 considers message $v_{2}$ sent by $p_{2}$ and received by $p_{3}$; again, process $p_{1}$ is the coordinator. Messages received from $p_{2}$ in round $3 \rho-2$ are not corrupted, and we show that the message received by $p_{3}$ from $p_{2}$ in macro-round $\rho$ can only be $v_{2}$ or $\perp$. In round $3 \rho$, process $p_{3}$ does not "blindly" adopt the value received from the coordinator (the message received can be corrupted). The value received in round $3 \rho$ from the coordinator is adopted by $p_{3}$ only if the same value is received from at least $f$ additional processes (line 23). This ensures that at least one such message is not corrupted. In Figure 3, process $p_{3}$ adopts $\perp$ as message received from $p_{2}$ in macro-round $\rho$, since it did not received $f+1$ messages equal to value $v_{2}^{\prime}$ received from the coordinator.

Lemma 1. If $n>3 f$ then Algorithm 1 preserves $\mathcal{P}_{\text {stat }}^{f}$.

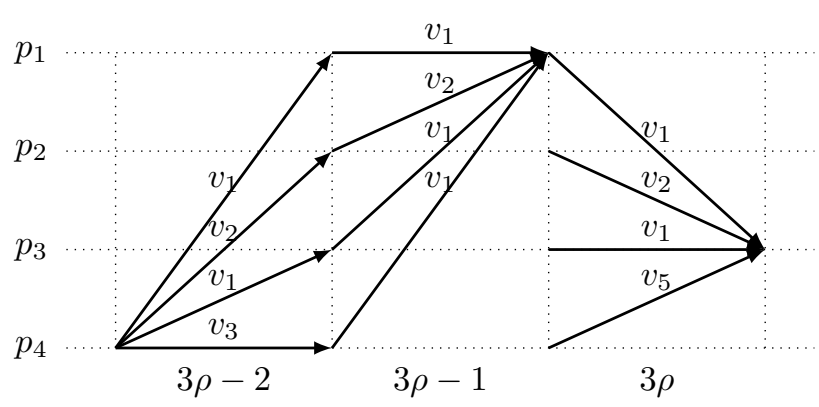

Figure 2: How Algorithm 1 ensures $\mathcal{P}_{\diamond \text { cons }}^{f}$ : point of view of message sent by $p_{4}$ and received by $p_{3}$. Process $p_{1}$ is the coordinator, $n=4, f=1$, only messages received from $p_{4}$ can be corrupted.

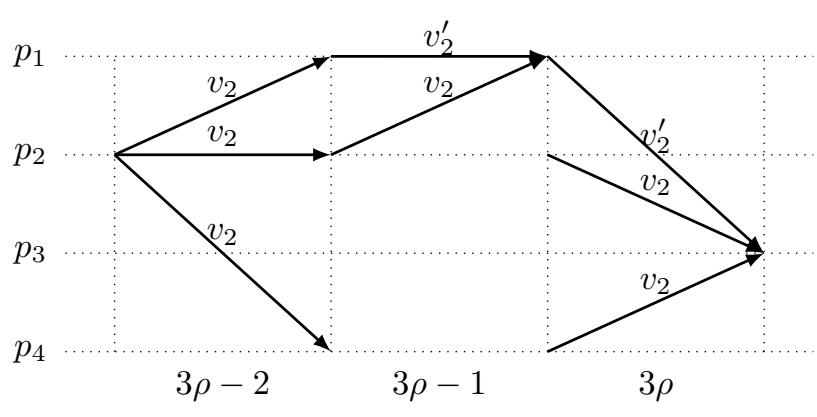

Figure 3: How Algorithm 1 preserves $\mathcal{P}_{\text {stat }}^{f}$ : point of view of $v_{2}$ sent by $p_{2}$ and received by $p_{3}$. Process $p_{1}$ is the coordinator, $n=4, f=1$, only messages received from $p_{1}$ can be corrupted. Absence of arrows represents message loss. Process $p_{3}$ can only receive $v_{2}$ or $\perp$ from $p_{2}$ (here $\perp)$.

Proof. To avoid ambiguities, let in this proof $A S_{\rho}=$ $\bigcup_{\rho>0} A S(\rho)$ denote the altered span with respect to macro-rounds implemented by Algorithm 1, while $A S=$ $\bigcup_{r>0} A S(r)$ denotes the altered span with respect to the rounds of Algorithm 1.

We need to show that $\left|A S_{\rho}\right| \leq f$ given that $|A S| \leq$ $f$. It is thus sufficient to show $A S_{\rho} \subseteq A S$. Assume by contradiction that there is a process $p \in \Pi$, a process $s \notin$ $A S$, and a macro-round $\rho$ so that $s \in A H O(p, \rho)$, i.e., $s$ sends message $m$ in macro-round $\rho$ and $p$ receives $m^{\prime} \neq$ $m$.

Then, because of line 23, for $Q=\left\{q: \vec{\mu}_{p}^{3 \rho}[q][s]=m^{\prime}\right\}$ we have $|Q| \geq f+1$. Because of $|A S| \leq f$, there is a $i \in Q$ that has received $i s]=m^{\prime}$. Moreover, this implies that $\vec{\mu}_{i}^{3 \rho-2}[s]=m^{\prime}$. Since $s$ sent $m$, this is a contradiction to $s \notin A S$. 
Lemma 2. If $n>3 f$ then Algorithm 1 simulates $\mathcal{P}_{\diamond \text { cons }}^{f}$ from $\mathcal{P}_{\diamond S K}^{f, 3(f+1)}$.

Proof. Let $\rho$ denote a macro-round, let $\Phi=$ $\{3 \rho-2,3 \rho-1,3 \rho\}$ be the set of rounds of $\rho$, and let $c_{0}=\rho \bmod n+1$ be the coordinator of $\rho$ such that

$$
c_{0} \in S K(\Phi) \wedge|S K(\Phi)| \geq n-f .
$$

Such a macro-round exists, because $(i) \mathcal{P}_{\diamond S K}^{f, 3(f+1)}$ holds and (ii) the coordinator is chosen using a rotating coordinator scheme (the coordinator of macro-round $\rho$ is process $\rho \bmod n+1$ ). We show that with Algorithm 1 (i) $\operatorname{CONS}(\rho)$ and (ii) $|S K(\rho)| \geq n-f$.

(i) Assume by contradiction that for two processes $p$ and $q, M s g_{p}^{(\rho)}$ and $M s g_{q}^{(\rho)}$ differ by the message of process $s \in \Pi$, that is $M s g_{p}^{(\rho)}[s] \neq M s g_{q}^{(\rho)}[s]$. By round $3 \rho$, every process adopts the value of $c_{0}$ or sets $\mathrm{Msg}^{(\rho)}[s]$ to $\perp$; when $c_{0} \in S K(\Phi)$ it follows that $M s g_{p}^{(\rho)}[s]$ or $M s g_{q}^{(\rho)}[s]$ is $\perp$. W.1.o.g. assume that $\operatorname{Msg}_{p}^{(\rho)}[s]=v$ and $M s g_{q}^{(\rho)}[s]=\perp$. For rounds $r \in[3 \rho-1,3 \rho]$, let

$$
R_{p}^{r}(v, s):=\left\{i \in \Pi: \vec{\mu}_{p}^{r}[i][s]=v\right\}
$$

represent the set of processes from which $p$ receives $v$ at position $s$ in round $\mathrm{r}$. Similarly, for rounds $r \in[3 \rho-$ $1,3 \rho]$, let

$$
Q^{r}(v, s):=\left\{i \in \Pi: S_{i}^{r}\left(s_{i}^{r}\right)[s]=v\right\}
$$

represent the set of processes that sent $v$ at position $s$ in round $\mathrm{r}$.

By line 23, if $\operatorname{Msg}_{p}^{(\rho)}[s]=v$, then $\left|R_{p}^{3 \rho}(v, s)\right| \geq f+1$, and $c_{0} \in R_{p}^{3 \rho}(v, s)$. Since $c_{0} \in S K(\Phi)$, we have $c_{0} \in$ $Q^{3 \rho}(v, s)$ and thus, by line $16,\left|R_{c_{0}}^{3 \rho-1}(v, s)\right| \geq 2 f+1$. From this and $|S K(\Phi)| \geq n-f$, we have $\mid R_{c_{0}}^{3 \rho-1}(v, s) \cap$ $S K(\Phi) \mid \geq f+1$. Therefore, at least $f+1$ processes $p^{\prime}$ in $S K(\Phi)$, including $c_{0}$, have received ${ }_{p^{\prime}}[s]=v$. It follows that $\left|R_{q}^{3 \rho}(v, s)\right| \geq f+1$, and $c_{0} \in R_{q}^{3 \rho}(v, s)$. This contradicts the assumption that the condition in line 23 is false for process $q$.

(ii) For every process $p \in \Pi$ and $q \in S K(\Phi)$, by definition we have received ${ }_{p}[q]=m_{q}$ at the end of round $3 \rho-2$. In round $3 \rho-1, c_{0}$ receives received $_{q^{\prime}}[q]=m_{q}$ from every process $q^{\prime} \in S K(\Phi)$, and thus there is no $q \in S K(\Phi)$ s.t. $\quad c_{0}$ sets received $c_{0}[q]$ to $\perp\left(^{*}\right)$. In round $3 \rho$, since $c_{0} \in S K(\Phi)$, every process $p$ receives the message from $c_{0}$. In addition, since $n>3 f$ and $|S K(\Phi)| \geq n-f$, every process receives the message from $n-f>f+1$ processes in $S K(\Phi)$. By (*) and line 23, for all processes $p$ and all $q \in S K(\Phi)$, we have $M s g_{p}[q]=m_{q}$. Thus $S K(\Phi) \subseteq$ $S K(\rho)$, which shows that $|S K(\rho)| \geq n-f$.
Corollary 1. If $n>3 f$, Algorithm 1 is a simulation of $\mathcal{P}_{\diamond \text { cons }}^{f} \wedge \mathcal{P}_{\text {stat }}^{f}$ from $\mathcal{P}_{\diamond S K}^{f, 3(f+1)} \wedge \mathcal{P}_{\text {stat }}^{f}$.

Remark 1. Algorithm 1 can easily be extended to preserve also the following predicate:

$$
|H O(p, r)| \geq n-f
$$

Intuitively, such an assumption is typical for algorithms that are designed to work in a system with reliable channels. The modified simulation algorithm then uses the reception vector of the first round as $M s g$ in case there would be less than $f$ elements in $M s g$. It is easy to show that this does not affect Corollary 1, while preserving the above predicate. Since our algorithms do not need this assumption, we do not detail this extension further.

Remark 2. Interestingly there is also decentralized (i.e., coordinator-free) solution to this simulation. The algorithm is presented in [8] in terms of Byzantine faults but can be easily adapted to our framework. Such a simulation requires $f+1$ rounds. In some cases this approach can be beneficial [7].

\subsection{Simulation in the presence of dynamic value faults}

In this section we show a simulation of $\mathcal{P}_{\diamond \text { cons }}$ from $\mathcal{P}_{\diamond S K}$ and the weaker predicate $\mathcal{P}_{d y n}$ that (partially) preserves $\mathcal{P}_{d y n}$. More precisely, we show a simulation from $\mathcal{P}_{\diamond S K}^{f} \wedge$ $\mathcal{P}_{d y n}^{\alpha}$ into $\mathcal{P}_{\diamond \text { cons }}^{f} \wedge \mathcal{P}_{d y n}^{\beta}$ with $\beta \geq \alpha$ : the simulation may only partially preserve $\mathcal{P}_{d y n}^{\alpha}$ in the sense that the number of corruptions in the simulated rounds may increase from $\alpha$ to $\beta \geq \alpha$, depending on $n$.

The simulation requires four rounds, as shown by Algorithm 2. As we can see, $\beta$ is not a parameter of the algorithm. Fixing $\beta$ leads to some requirement on $n$. More precisely, given $f, \alpha \geq f, \beta \geq \alpha$, Algorithm 2 requires $n>\frac{(\beta+1)(\alpha+f)}{\beta-\alpha+1}$. Similarly to Algorithm 1, it is coordinator-based.

The communication pattern of Algorithm 2 is very similar to Algorithm 1 with the addition of one "all-to-all" round (see Figure 4, to be compared with Figure 1). We explain Algorithm 2 from the point of view of the message sent by process $p_{2}$. In round $4 \rho-3$, process $p_{2}$ sends message $v_{2}$ to all. ${ }^{9}$ In round $4 \rho-2$, all processes send to all the value received from $p_{2}$, and then compare the value

\footnotetext{
${ }^{9}$ Similar as in the description of Algorithm 1, in case of messages that contain a vector of messages, we focus only on those elements of the vectors that are related to the message sent by process $p_{2}$
} 


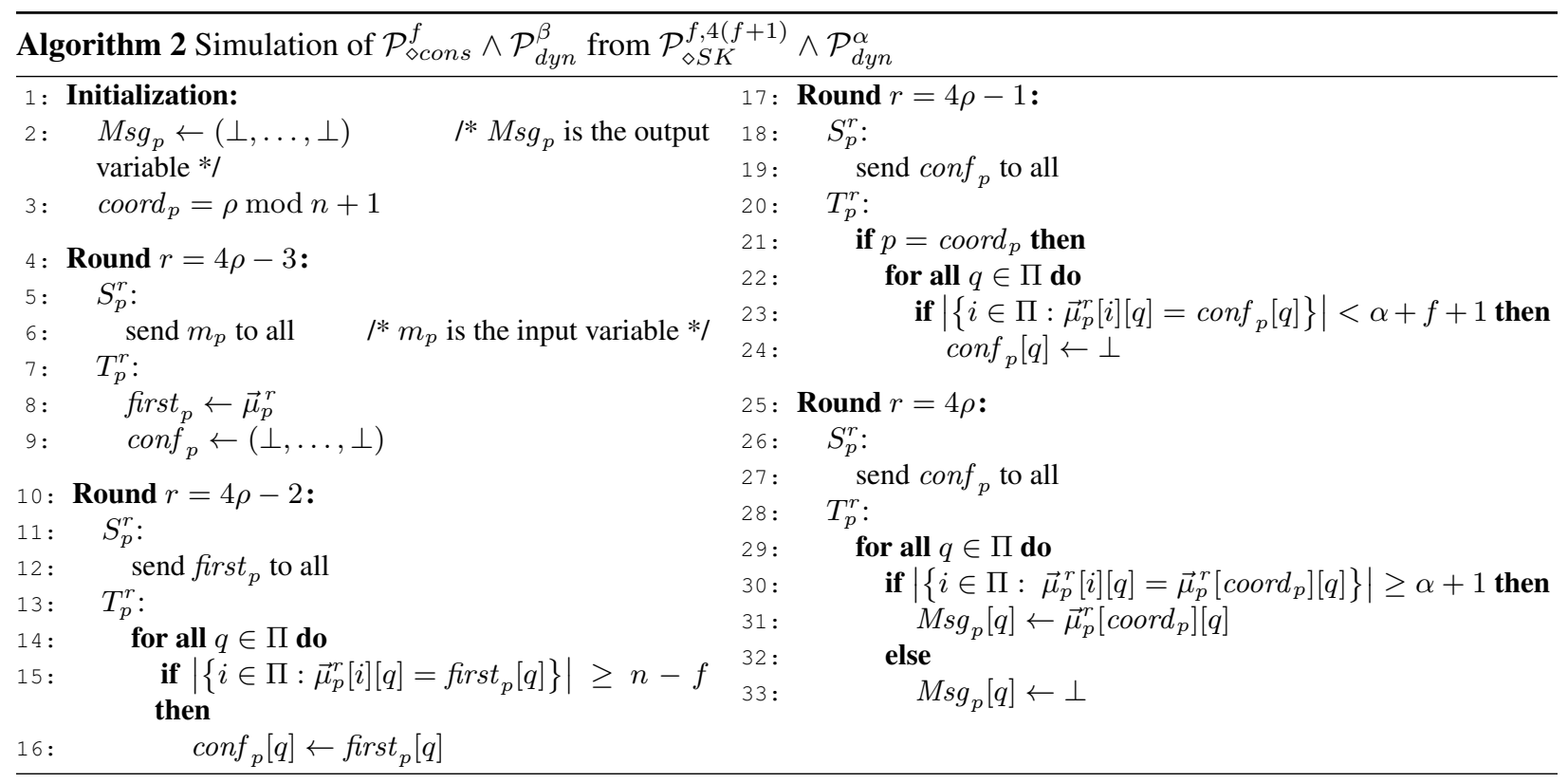

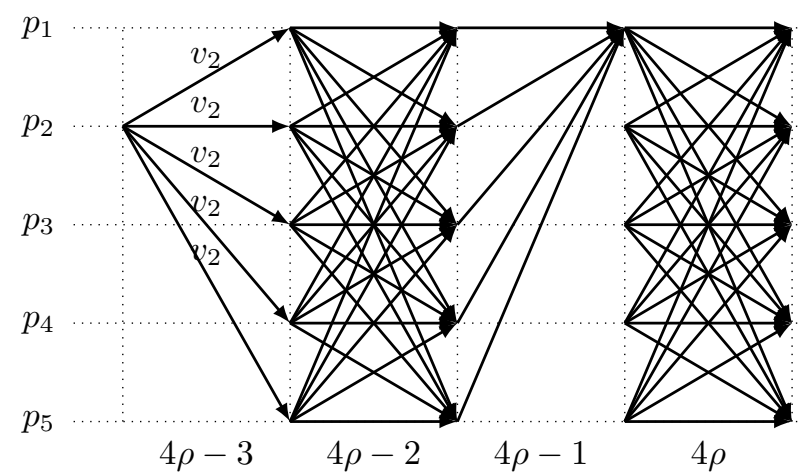

Figure 4: Algorithm 2 from the point of view of $v_{2}$ sent by $p_{2} ; p_{1}$ is the coordinator, $n=4, f=1$.

$v_{2}$ received from $p_{2}$ in round $4 \rho-3$ with the value indirectly received from the other processes in round $4 \rho-2$. If at least $n-f$ values $v_{2}$ have been received by process $p$, then $p$ keeps $v_{2}$ as the message received from $p_{2}$. Otherwise, the message received from $p_{2}$ is $\perp$ (line 9). As explained later, rounds $4 \rho-3$ and $4 \rho-2$ filter the values for rounds $4 \rho-1$ and $4 \rho$ in order to ensure $\mathcal{P}_{d y n}^{\beta}$ from $\mathcal{P}_{d y n}^{\alpha}$. Rounds $4 \rho-1$ and $4 \rho$ are very similar to rounds $3 \rho-1$ and $3 \rho$ in Algorithm 1 .

Algorithm 2 relies on a coordinator for ensuring $\mathcal{P}_{\diamond \text { cons }}^{f}$ : all processes assign to $M s g_{p}$ the value received from the coordinator in round $4 \rho$ (see line 31 ). This is achieved during a macro-round in which the size of the

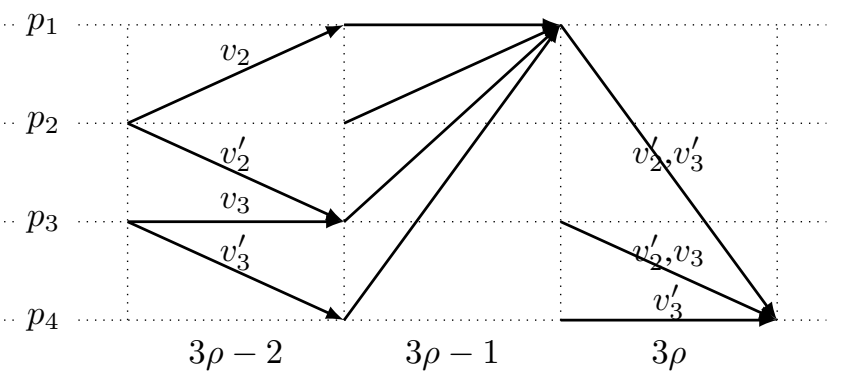

Figure 5: Algorithm 1 does not preserves $\mathcal{P}_{d y n}^{\alpha}$; from the point of view of $p_{2}$ and $p_{3}$, that sends correspondingly $v_{2}$ and $v_{3}$ in round $3 \rho-2$, and reception of process $p_{4}$ of messages sent by $p_{2}$ and $p_{3} . n=4, f=\alpha=\beta=$ 1 and $p_{1}$ is coordinator. Message received by $p_{4}$ from coordinator in round $3 \rho$ is corrupted; other messages are correctly received. Absence of arrows represents message loss.

safe kernel is at least $n-f$, with the coordinator in the safe kernel. Since consistency is ensured under the same conditions as with Algorithm 1, we use exactly the same mechanism in Algorithm 2.

The additional complexity of Algorithm 2 comes from the part responsible for ensuring $\mathcal{P}_{d y n}^{\beta}$. We start by explaining on Figure 5 why Algorithm 1 does not preserve $\mathcal{P}_{d y n}^{\alpha}$ for the simplest case $f=\alpha=1, n=4$. According to $\mathcal{P}_{d y n}^{1}$, every process can receive at most one 
corrupted message per round. In round $3 \rho-2$, process $p_{3}$ receives the corrupted message $v_{2}^{\prime}$ from $p_{2}$, and $p_{4}$ receives the corrupted value $v_{3}^{\prime}$ from $p_{3}$. These values are sent to the coordinator $p_{1}$ in round $3 \rho-1$. Finally, in round $3 \rho$, process $p_{4}$ receives $v_{2}^{\prime}, v_{3}^{\prime}$ from $p_{1}, v_{2}^{\prime}, v_{3}$ from $p_{3}$, and $v_{3}^{\prime}$ from itself. Since there are $f+1$ values equal to those sent by coordinator, $p_{4}$ considers $v_{2}^{\prime}$, respect. $v_{3}^{\prime}$, as messages received from $p_{2}$, respect. $p_{3}$, in macro-round $\rho$, violating $\mathcal{P}_{d y n}^{1}$. The problem comes from the fact that dynamic faults have a cumulative effect, i.e., messages that are corrupted in round $3 \rho-2$ add to corrupted messages from round $3 \rho$.

We now explain why the addition of round $4 \rho-2$ allows us to cope with this issue. Informally speaking, the role of round $4 \rho-2$ in Algorithm 2 is to transform dynamic faults into some maximum number of static faults, i.e., into some maximum number of faults localized at some fixed set of processes. Consider rounds $4 \rho-3$ and $4 \rho-2$, with $n=4, \alpha=f=1$. In round $4 \rho-3$, predicate $\mathcal{P}_{d y n}^{\alpha}$ ensures that, in total, at most $n \cdot \alpha=4$ corrupted values are received. In other words, among the vectors first $_{p_{1}}$ to first $_{p_{4}}$ received (line 8), at most $n \cdot \alpha=4$ elements can be corrupted (see Figure 6 , where $\times$ represents possible corrupted values). In round $4 \rho-2$, each process $p_{i}$ sends vector first $_{p_{i}}$ to all processes. Consider the reception of these four vectors by some process $p_{j}$. Since $\alpha=1$, one of these vectors can be received corrupted at $p_{j}$. Figure 7 shows four examples, two starting from Figure 6 left, two starting from Figure 6 right.

To understand which value $p$ adopts from $q$ (lines 15 and 16) we need to look at column $q$ in Figure 7. From line 16, $p$ adopts a corrupted value from $q$ only if column $q$ contains at least $n-f=3$ corrupted values. In the upper case, no column satisfies this condition, i.e., $p$ adopts no corrupted value. In the lower case, columns 2 and 1 satisfy this condition, i.e., corrupted values can be adopted from $p_{2}$ or $p_{1}$. It is easy to see that in the case $n=4, f=$ $\alpha=1$, corrupted values can be adopted from at most one process. In other words, rounds $4 \rho-3$ and $4 \rho-2$ has transformed $\alpha=1$ dynamic fault into at most $\beta=1$ static faults. However, in the case $n=5, f=\alpha=2$, rounds $4 \rho-3$ and $4 \rho-2$ transform $\alpha=2$ dynamic fault into at most $\beta=3$ static fault.

Transforming $\alpha$ dynamic faults into $\beta \geq \alpha$ static faults allows us to rely on the same mechanism as in Algorithm 1 for the last two rounds of the simulation. Note that in rounds $4 \rho-1$ and $4 \rho$ of Algorithm 2 we have dynamic faults, while in rounds $3 \rho-1$ and $3 \rho$ of Algorithm 1 faults were static. Nevertheless the same mechanisms can be used in both cases.

Theorem 1. If $n>\frac{(\beta+1)(\alpha+f)}{\beta-\alpha+1}, n>\alpha+f, \alpha \geq f$, and
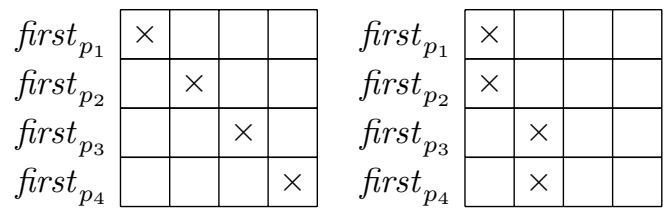

Figure 6: After round $4 \rho-3$ : Two examples (left and right) of corrupted values (represented by $\mathrm{X}$ ).
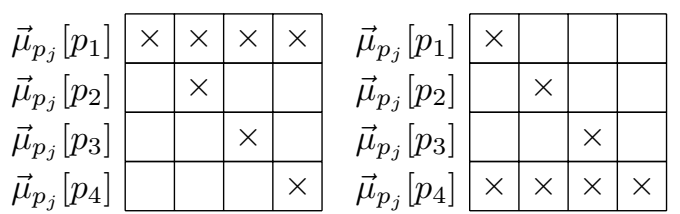

(a)
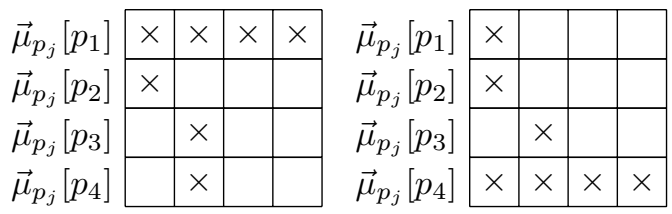

(b)

Figure 7: After round $4 \rho-2$ : (a) Two examples of vectors received by some $p_{j}$ starting from Fig. 6 left; (b) Two examples of vectors received by some $p_{j}$ starting from Fig. 6 right (corrupted values are represented by $\times$ ).

$\beta \geq \alpha$, then Algorithm 2 simulates $\mathcal{P}_{\diamond \text { cons }}^{f} \wedge \mathcal{P}_{\text {dyn }}^{\beta}$ from $\mathcal{P}_{\diamond S K}^{f, 4(f+1)} \wedge \mathcal{P}_{d y n}^{\alpha}$.

The theorem follows directly from Lemmas 3 and 4: the first lemma considers $\mathcal{P}_{d y n}^{\beta}$ and $\mathcal{P}_{d y n}^{\alpha}$, the second $\mathcal{P}_{\diamond \text { cons }}^{f}$ and $\mathcal{P}_{\diamond S K}^{f, 4(f+1)}$.

Lemma 3. If $n>\frac{(\beta+1)(\alpha+f)}{\beta-\alpha+1}$ and $\beta \geq \alpha$, then Algorithm 2 simulates $\mathcal{P}_{\text {dyn }}^{\beta}$ from $\mathcal{P}_{\text {dyn }}^{\alpha}$.

Proof. We need to show that for every macro-round $\rho$, and every process $p$, we have $|A H O(p, \rho)| \leq \beta$, i.e., at most $\beta$ messages are corrupted.

Assume by contradiction that there is a process $p$ so that $|A H O(p, \rho)|>\beta$. That is, we have $|S| \geq \beta+1$ for

$$
S=\left\{s \in \Pi: M_{s g_{p}}[s] \neq m_{s} \text { and } M_{s g_{p}}[s] \neq \perp\right\}
$$

For all $s \in S$, let $m_{s}^{\prime}$ denote $\operatorname{Msg}_{p}[s]$. The output $\operatorname{Msg}_{p}[s]$ is set at line 31. Because of line 30, this implies that

$$
\forall s \in S:\left|\left\{i \in \Pi: \vec{\mu}_{p}^{4 \rho}[i][s]=m_{s}^{\prime}\right\}\right| \geq \alpha+1 .
$$

Because of $|A H O(p, 4 \rho)| \leq \alpha$, at the end of round $4 \rho-1$ we have

$$
\forall s \in S, \exists i_{s} \in \Pi: \operatorname{conf}_{i_{s}}[s]=m_{s}^{\prime} .
$$


Since in round $4 \rho-1$ the elements of conf can only be set to $\perp$, the same condition needs to holds also at the end of round $4 \rho-2$. Because of line 15, this implies

$$
\begin{gathered}
\forall s \in S, \exists i_{s} \in \Pi, \exists Q_{s} \subseteq \Pi,\left|Q_{s}\right| \geq n-f, \forall q \in Q_{s}: \\
\vec{\mu}_{i_{s}}^{4 \rho-2}[q][s]=m_{s}^{\prime} .
\end{gathered}
$$

Because of $|A H O(p, 2)| \leq \alpha$, at the end of round $4 \rho-3$ we have

$$
\begin{gathered}
\forall s \in S, \exists Q_{s}^{\prime} \subseteq \Pi,\left|Q_{s}^{\prime}\right| \geq n-f-\alpha: \forall q \in Q_{s}^{\prime}: \\
\text { first }_{q}[s]=m_{s}^{\prime} .
\end{gathered}
$$

Note that frst $_{q}=\vec{\mu}_{q}^{4 \rho-3}$. The number of tuples $(q, s)$ such that $\vec{\mu}_{q}^{4 \rho-3}[s]=m_{s}^{\prime}$ is thus at least $(\beta+1)(n-f-\alpha)$. From this it follows that there is at least one process $q_{0}$ where the number of corrupted messages in the first round is

$\left\lceil\frac{(\beta+1)(n-f-\alpha)}{n}\right\rceil=\alpha+\left\lceil\frac{(\beta+1)(n-f-\alpha)-n \alpha}{n}\right\rceil$

where the last inequation follows from $n>\frac{(\beta+1)(\alpha+f)}{\beta-\alpha+1}$ and $\beta \geq \alpha$, which ensures $(\beta+1)(n-f-\alpha)-n \alpha>0$. Therefore $A H O\left(q_{0}, 4 \rho-3\right)>\alpha$, which contradicts the assumption $\mathrm{AHO}\left(q_{0}, 4 \rho-3\right) \leq \alpha$.

Lemma 4. If $n>\alpha+f$ and $\alpha \geq f$, then Algorithm 2 simulates $\mathcal{P}_{\diamond \text { cons }}^{f}$ from $\mathcal{P}_{\diamond S K}^{f, 4(f+1)}$.

Proof. Let $\rho$ denote a macro-round, let $\Phi=$ $\{4 \rho-3, \ldots, 4 \rho\}$ be the set of rounds of $\rho$, and let $c_{0}=\rho \bmod n+1$ be the coordinator of $\rho$ such that

$$
c_{0} \in S K(\Phi) \wedge|S K(\Phi)| \geq n-f
$$

Such a macro-round exists, because $(i) \mathcal{P}_{\diamond S K}^{f, 4(f+1)}$ holds and (ii) the coordinator is chosen using a rotating coordinator scheme (the coordinator of macro-round $\rho$ is process $\rho \bmod n+1)$. We show that with Algorithm 2 (i) $\operatorname{CONS}(\rho)$ and (ii) $|S K(\rho)| \geq n-f$.

(i) Assume by contradiction that for two processes $p$ and $q, M s g_{p}^{(\rho)}$ and $M s g_{q}^{(\rho)}$ differ by the message of process $s \in \Pi$, that is $M s g_{p}^{(\rho)}[s] \neq M s g_{q}^{(\rho)}[s]$. By round $4 \rho$, every process adopts the value of $c_{0}$ or sets $\operatorname{Msg}^{(\rho)}[s]$ to $\perp$; when $c_{0} \in S K(\Phi)$ it follows that $M s g_{p}^{(\rho)}[s]$ or $M s g_{q}^{(\rho)}[s]$ is $\perp$. W.1.o.g. assume that $\operatorname{Msg}_{p}^{(\rho)}[s]=v$ and $M s g_{q}^{(\rho)}[s]=\perp$. For rounds $r \in[4 \rho-1,4 \rho]$, let $R_{p}^{r}(v, s):=\left\{q \in \Pi: \vec{\mu}_{p}^{r}[q][s]=v\right\}$ represent the set of processes from which $p$ receives $v$ at position $s$. Similarly, for rounds $r \in[4 \rho-1,4 \rho]$, let

$$
Q^{r}(v, s):=\left\{q \in \Pi: S_{q}^{r}\left(s_{q}^{r}\right)[s]=v\right\}
$$

represent the set of processes that sent $v$ at position $s$.

By line 30, if $M s g_{p}^{(\rho)}[s]=v$, then $\left|R_{p}^{4 \rho}(v, s)\right| \geq \alpha+1$, and $c_{0} \in R_{p}^{4 \rho}(v, s)$. Since $c_{0} \in S K(\Phi)$, we have $c_{0} \in$ $Q^{4 \rho}(v, s)$ and thus, by line $23,\left|R_{c_{0}}^{4 \rho-1}(v, s)\right| \geq \alpha+f+1$. From this and $|S K(\Phi)| \geq n-f$, we have $\mid R_{c_{0}}^{4 \rho-1}(v, s) \cap$ $S K(\Phi) \mid \geq \alpha+1$. Therefore, at least $\alpha+1$ processes $p^{\prime}$ in $S K(\Phi)$, including $c_{0}$, have $\operatorname{conf}_{p^{\prime}}[s]=v$. It follows that $\left|R_{q}^{4 \rho}(v, s)\right| \geq \alpha+1$, and $c_{0} \in R_{q}^{4 \rho}(v, s)$. This contradicts the assumption that the condition in line 30 is false for process $q$.

(ii) For every processes $p \in \Pi$ and $q \in S K(\Phi)$, by

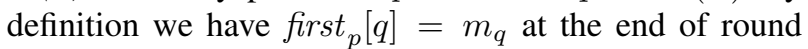
$4 \rho-3$. In round $4 \rho-2$, for every process $s \in S K(\Phi)$, first $_{s}$ is received. Therefore, by line 15 since $|S K(\Phi)| \geq$ $n-f$, at every process $p \in \Pi$ we have $\operatorname{conf} f_{p}[q]=m_{q}$, for all $q \in S K(\Phi)(*)$. In round $4 \rho-1, c_{0}$ receives conf $_{q^{\prime}}[q]=m_{q}$ from every process $q^{\prime} \in S K(\Phi)$, and thus there is no $q \in S K(\Phi)$ s.t. $c_{0}$ sets $\operatorname{conf}_{c_{0}}[q]$ to $\perp$ (**). In round $4 \rho$, since $c_{0} \in S K(\Phi)$, every process $p$ receives $>$ the message from $c_{0}$. In addition, since $n \geq f+\alpha+1$ and $|S K(\Phi)| \geq n-f$, every process receives the message from $n-f \geq \alpha+1$ processes in $S K(\Phi)$. By (*), (**) and line 30, for all processes $p$ and all $q \in S K(\Phi)$, we have $M s g_{p}[q]=m_{q}$. Thus $S K(\Phi) \subseteq S K(\rho)$, which shows that $S K(\rho) \geq n-f$.

Corollaries 2 and 3 follow from Lemma 3.

Corollary 2. If $n>(\alpha+1)(\alpha+f)$, then Algorithm 2 preserves $\mathcal{P}_{d y n}^{\alpha}$.

By Corollary 2, preserving $\mathcal{P}_{d y n}^{\alpha}$ leads to a quadratic dependency between $n$ and $\alpha$. Corollary 3 shows the surprising result that, allowing more than $\alpha$ corruptions in the simulated round, leads instead to a linear dependency between $n$ and $\alpha$. Note that the simulation mentioned in Corollary 3 is not useful if $\left[\frac{\eta}{\eta-1} \alpha\right] \geq n$.

Corollary 3. For any $\eta \in \mathbb{R}, \eta>1$, if $n>\eta(\alpha+f)$, then Algorithm 2 simulates $\mathcal{P}_{d y n}^{\left\lfloor\frac{\eta}{\eta-1} \alpha\right\rfloor}$ from $\mathcal{P}_{d y n}^{\alpha}$.

Proof. Let $\xi=\frac{\eta}{\eta-1}$. From $\lfloor\xi \alpha\rfloor>\xi \alpha-1=\alpha \frac{\eta}{\eta-1}-$ $1=\frac{\alpha \eta-\eta+1}{\eta-1}$ it follows that $\frac{\lfloor\xi \alpha\rfloor+1}{\lfloor\xi \alpha\rfloor-\alpha+1}<\eta$. The corollary follows from Lemma 3 by setting $\beta=\lfloor\xi \alpha\rfloor$.

\subsection{Generic predicate}

In Section 6 we solve consensus using the following generic predicate, which combines $\mathcal{P}_{\diamond \text { cons }}$ and $\mathcal{P}_{\diamond S K}$ :

$$
\begin{aligned}
\mathcal{P}_{\diamond \text { cons } \oplus S K}^{f, b, k}: & : \forall \phi>0, \exists \phi_{0} \geq \phi, \\
& \operatorname{CONS}\left(\left(\phi_{0}-1\right) k+1\right) \wedge|S K(\Phi)| \geq n-f, \\
& \text { where } \Phi=\left\{\left(\phi_{0}-1\right) k+1-b, \ldots, \phi_{0} k\right\}
\end{aligned}
$$


It defines a phase with $k$ rounds, where the first round of some phase $\phi_{0}$ is consistent and all rounds of phase $\phi_{0}$ plus the preceding $b$ rounds have safe kernel of size at least equal to $n-f$.

Obviously, $\mathcal{P}_{\diamond \text { cons } \oplus S K}$ can be simulated from $\mathcal{P}_{\diamond S K}$ and $\mathcal{P}_{\text {stat }}$ using Algorithm 1, and from $\mathcal{P}_{\diamond S K}$ and $\mathcal{P}_{d y n}$ using Algorithm 2. In both cases, Algorithm 1 or Algorithm 2 simulate the first round of a phase, and a trivial simulation (where messages are just delivered as received) are used for the other rounds. Ensuring that the coordinator is in the safe kernel requires $f+1$ phases. In case of Algorithm 1, the first macro-round of a phase requires 3 rounds, and the others $k-1$ only 1 round. Therefore $f+1$ phases correspond to $(k+2)(f+1)$ rounds. This leads to:

Corollary 4. If $n>3 f$, then $\mathcal{P}_{\diamond \text { cons } \oplus S K}^{f, b, k} \wedge \mathcal{P}_{\text {stat }}^{f}$ can be simulated from $\mathcal{P}_{\diamond S K}^{f, K} \wedge \mathcal{P}_{\text {stat }}^{f}$, where $K=(k+2)(f+$ $1)+b+(k+1)$.

Note that the additional term $k+1$ for $K$ stems from the fact that the rounds with a safe kernel are not necessarily aligned to the phases of $\mathcal{P}_{\diamond \text { cons }}^{f, b, k}$. In case of Algorithm 2 , since the first macro-round requires 4 rounds, we have:

Corollary 5. If $n>\frac{(\beta+1)(\alpha+f)}{\beta-\alpha+1}, n>\alpha+f, \alpha \geq f$, and $\beta \geq \alpha$, then $\mathcal{P}_{\diamond \text { cons } \oplus S K}^{f, b, k} \wedge \mathcal{P}_{\text {dyn }}^{\beta}$ can be simulated from $\mathcal{P}_{\diamond S K}^{f, K} \wedge \mathcal{P}_{d y n}^{\alpha}$, where $K=(k+3)(f+1)+b+(k+2)$.

Here the additional alignment term in $K$ is $k+2$.

\section{Solving consensus with eventual consistency}

In this section we use the generic predicate $\mathcal{P}_{\diamond \text { cons } \oplus S K}$ to solve consensus. In all consensus algorithms below, the notation $\#(v)$ is used to denote the number of messages received with value $v$, i.e.,

$$
\#(v) \equiv\left|\left\{q \in \Pi: \vec{\mu}_{p}^{r}[q]=v\right\}\right|
$$

\subsection{The $B O T R$ algorithm}

We start with the simplest algorithm, namely the BOT $R$ algorithm. The basic technique of this algorithm is that a value that is decided is locked in the sense that a sufficiently high quorum of processes retain this value as estimate. A similar algorithmic scheme can be found in

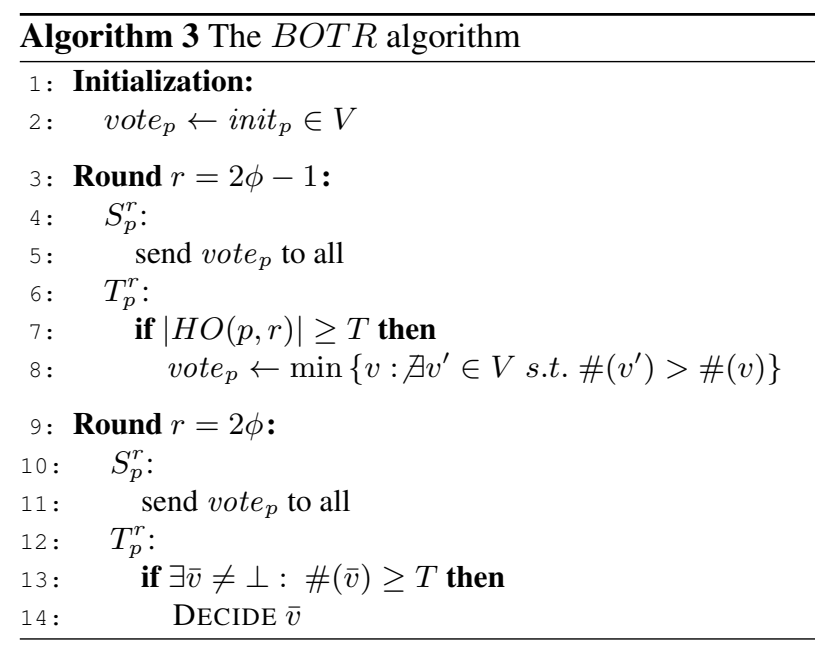

algorithms for benign $[9,24,16,11]$ and arbitrary $[20,5]$ faults.

The code of BOTR is given as Algorithm 3. It consists of a sequence of phases, where each phase $\phi$ has two rounds $2 \phi-1$ and $2 \phi$. Every process $p$ maintains a single variable $v^{\circ} e_{p}$ initialized to $p$ 's initial value. In every round, every process $p$ sends vote $_{p}$ to all. In round $2 \phi-1$, if a process $p$ receives at least $T$ messages then it updates vote $_{p}$, and sets vote $_{p}$ to the smallest most often received value of the current round. In round $2 \phi$, if a process $p$ receives at least $T$ times the same value $v$ then it decides on $v{ }^{10}$

We will show that BOTR is safe (in the sense that it fulfills integrity and agreement) for appropriate choices of $T$ when $\mathcal{P}_{d y n}^{\alpha}$ holds (or $\mathcal{P}_{\text {stat }}^{\alpha}$, since $\mathcal{P}_{\text {stat }}^{\alpha}$ implies $\mathcal{P}_{d y n}^{\alpha}$ ). The value of threshold $T$ is chosen such that if some process decides $v$ at line 14 of round $r$, then in any round $r^{\prime} \geq r$, at all processes only $v$ can be assigned to any vote, and hence only $v$ can be decided. Termination is achieved in both cases if in addition the following predicate holds:

$$
\begin{aligned}
\mathcal{P}_{\text {BOTR }}^{f}:: & \forall \phi, \forall p, \exists \phi_{0}>\phi: \\
& C O N S\left(2 \phi_{0}-1\right) \wedge\left|H O\left(p, 2 \phi_{0}-1\right)\right| \geq n-f \\
& \wedge\left(\exists \phi_{1} \geq \phi_{0}:\left|\operatorname{SHO}\left(p, 2 \phi_{1}\right)\right| \geq n-f\right)
\end{aligned}
$$

Obviously, $\mathcal{P}_{\diamond \text { cons } \oplus S K}^{f, 0,2}$ implies $\mathcal{P}_{B O T R}^{f}$. Eventual consistency ensures the first part of the predicate, namely the existence of a consistent round $2 \phi_{0}-1$ where in addition every process receives enough messages. This guarantees that at the end of round $2 \phi_{0}-1$ all processes adopt

\footnotetext{
${ }^{10}$ The two rounds of BOT $R$ algorithm can be merged in a single round in which the code of both state-transition functions is executed at once. We have split them in two rounds to emphasize on the different communication predicates required.
} 
the same value for vote $_{p}$. The second part of the predicate forces every process to make a decision at the end of round $2 \phi_{1}$.

\subsubsection{Correctness of the $B O T R$ algorithm}

First we introduce some piece of notation. For any variable $x$ local to process $p$, we denote $x_{p}^{(r)}$ the value of $x_{p}$ at the end of round $r$. For any value $v \in V$ and any process $p$, at any round $r>0$, we define the sets $R_{p}^{r}(v)$ and $Q_{p}^{r}(v)$ as follows:

$$
\begin{aligned}
& R_{p}^{r}(v):=\left\{q \in \Pi: \vec{\mu}_{p}^{r}[q]=v\right\} \\
& Q_{p}^{r}(v):=\left\{q \in \Pi: S_{q}^{r}\left(p, s_{q}\right)=v\right\} .
\end{aligned}
$$

where $s_{q}$ denotes $q$ 's state at the beginning of round $r$. The set $R_{p}^{r}(v)$ (resp. $Q_{p}^{r}(v)$ ) represents the set of processes from which $p$ receives $v$ (resp. which ought to send $v$ to $p$ ) at round $r$. Since at each round of the consensus algorithm, every process sends the same message to all, the sets $Q_{p}^{r}(v)$ do not depend on $p$, and so can be just denoted by $Q^{r}(v)$ without any ambiguity.

We start our correctness proof with a general basic lemma:

Lemma 5. For any process $p$ and any value $v$, at any round $r$, we have:

$$
\left|R_{p}^{r}(v)\right| \leq\left|Q^{r}(v)\right|+|A H O(p, r)|
$$

Proof. Suppose that process $p$ receives a message with value $v$ at round $r>0$ from process $q$. Then, either the code of $q$ prescribes it to send $v$ to $p$ at round $r$, i.e., $q$ belongs to $Q^{r}(v)$ and thus $q$ is also in $\operatorname{SHO}(p, r)$, or the message has been corrupted and $q$ is in $A H O(p, r)$. It follows that $R_{p}^{r}(v) \subseteq Q^{r}(v) \cup A H O(p, r)$, which implies $\left|R_{p}^{r}(v)\right| \leq\left|Q^{r}(v)\right|+|A H O(p, r)|$.

As an intermediate step to argue agreement, our next lemma introduces a condition on $T$ that ensures no two processes can decide differently at the same round:

Lemma 6. If $T>\frac{n}{2}+\alpha$ then in any run of the $\mathrm{HO}$ machine $\left\langle B O T R, \mathcal{P}_{d y n}^{\alpha}\right\rangle$ there is at most one possible decision value per round.

Proof. Assume by contradiction that there exist two processes $p$ and $q$ that decide on different values $v$ and $v^{\prime}$ in some round $r>0$. From the code of BOTR, we deduce that $\left|R_{p}^{r}(v)\right| \geq T$ and $\left|R_{q}^{r}\left(v^{\prime}\right)\right| \geq T$. Then Lemma 5 ensures that $\left|Q^{r}(v)\right| \geq T-|A H O(p, r)|$ and $\left|Q^{r}\left(v^{\prime}\right)\right| \geq T-|A H O(q, r)|$.

Since each process sends the same value to all at each round $r$, the sets $Q^{r}(v)$ and $Q^{r}\left(v^{\prime}\right)$ are disjoint if $v$ and $v^{\prime}$ are distinct values. Hence $\left|Q^{r}(v) \cup Q^{r}\left(v^{\prime}\right)\right|=\left|Q^{r}(v)\right|+$ $\left|Q^{r}\left(v^{\prime}\right)\right|$. Then from $T>\frac{n}{2}+\alpha$ and since $\mathcal{P}_{d y n}^{\alpha}$ holds, we have $|A H O(p, r)| \leq \alpha$ and $|A H O(q, r)| \leq \alpha$. Therefore, we derive that $\left|Q^{r}(v) \cup Q^{r}\left(v^{\prime}\right)\right|>2(T-\alpha)>n$, a contradiction.

The next lemma shows that once a sufficently high number of processes have the same vote, no other value will be adopted in later rounds by any process:

Lemma 7. If $T>\frac{2}{3}(n+2 \alpha)$, then in any run of the HO machine $\left\langle B O T R, \mathcal{P}_{d y n}^{\alpha}\right\rangle$, if $\mid\left\{p^{\prime} \in \Pi: \operatorname{vot}_{p^{\prime}}^{(r-1)}=\right.$ $v\} \mid \geq T-\alpha$, every process $q$ that updates its variable vote $_{q}$ at round $r$ sets it to $v$.

Proof. Since vote is only updated in the first round of a phase, it sufficies to consider the case $r=2 \phi-1$. Since $q$ updates vote $_{q}$ in round $r$, because of line 13, $|H O(q, r)| \geq T$. Let $Q^{r}(\bar{v})$ denote the set of processes that, according to their sending functions, ought to send messages different from $v$ at round $r$, and let $R_{q}^{r}(\bar{v})$ denote the set of processes from which $q$ receives values different from $v$ at round $r$. Since each process sends a message to all at each round, $Q^{r}(\bar{v})=\Pi \backslash Q^{r}(v)$, and thus $\left|Q^{r}(\bar{v})\right|=n-\left|Q^{r}(v)\right|$. Similarly, we have $R_{q}^{r}(\bar{v})=H O(q, r) \backslash R_{q}^{r}(v)$, and since $R_{q}^{r}(v) \subseteq H O(q, r)$, it follows that $\left|R_{q}^{r}(\bar{v})\right| \leq T-R_{q}^{r}(v)$.

Because of the assumption of the Lemma, and the fact that processes send their current value of vote in every round, we have $\left|Q^{r}(v)\right| \geq T-\alpha$. It follows that $\left|Q^{r}(\bar{v})\right| \leq n-(T-\alpha)$. With an argument similar to the one used in the proof of Lemma 5, we derive that $\left|R_{q}^{r}(\bar{v})\right| \leq\left|Q^{r}(\bar{v})\right|+|A H O(q, r)|$. Therefore, we obtain $\left|R_{q}^{r}(\bar{v})\right| \leq n-T+\alpha+|A H O(q, r)|$.

Since $\mathcal{P}_{d y n}^{\alpha}$ holds, it follows that $\left|R_{q}^{r}(\bar{v})\right| \leq n-T+$ $2 \alpha$. It follows that because of $T>\frac{2}{3}(n+2 \alpha)$, we have $\left|R_{q}^{r}(\bar{v})\right|<\frac{1}{3}(n+2 \alpha)$, and therefore $\left|R_{q}^{r}(v)\right|>\left|R_{q}^{r}(\bar{v})\right|$. This implies that $v$ is the most frequent value received by $q$ at round $r$. Then the code entails $q$ to set vote $q$ to $v$.

We now extend the statement of Lemma 7 to hold also for any phase $\phi>\phi_{0}$ :

Lemma 8. If $T>\frac{2}{3}(n+2 \alpha)$, then in any run of the HO machine $\left\langle B O T R, \mathcal{P}_{d y n}^{\alpha}\right\rangle$ such that process $p$ decides some value $v$ at some phase $\phi_{0}>0$, every process $q$ that updates its variable vote vat $_{\text {some }}$ ahase $\phi>\phi_{0}$ necessarily sets it to $v$.

Proof. Assume process $p$ decides value $v$ at round $r_{0}=$ $2 \phi_{0}$ of phase $\phi_{0}$. We prove by induction on $r$ that:

$$
\forall r \geq r_{0},\left|\left\{q \in \Pi: \operatorname{vote}_{q}^{(r-1)}=v\right\}\right| \geq T-\alpha .
$$


Then Lemma 7 ensures this Lemma.

Basic case: $r=r_{0}$. Since $p$ decides $v$ at round $r_{0}$, then $\left|R_{p}^{r_{0}}(v)\right| \geq T$. By Lemma 5, we have $\left|Q^{r_{0}}(v)\right| \geq T-\alpha$ when $\mathcal{P}_{d y n}^{\alpha}$ holds. From the code of BOTR, we have $Q^{r_{0}}(v)=\left\{q \in \Pi: \operatorname{vote}_{q}^{\left(r_{0}-1\right)}=v\right\}$, and so the basic case follows.

Inductive step: $r>r_{0}$. Assume $\mid\{q \in \Pi$ : $\left.\operatorname{vote}_{q}^{(r-1)}=v\right\} \mid \geq T-\alpha$. Lemma 7 ensures that for any process $q$, vote $q$ is updated only to $v$. Thus $\left|\left\{q \in \Pi: \operatorname{vote}_{q}^{(r)}=v\right\}\right| \geq \mid\left\{q \in \Pi: \operatorname{vote}_{q}^{(r-1)}=\right.$ $v\} \mid \geq T-\alpha$.

With the help of the previous lemmas, we can show the agreement clause of consensus:

proposition 1 (Agreement). If $T>\frac{2}{3}(n+2 \alpha)$, then there is at most one possible decision value in any run of the $\mathrm{HO}$ machine $\left\langle B O T R, \mathcal{P}_{d y n}^{\alpha}\right\rangle$.

Proof. Let $\phi_{0}$ be the first phase at which some process $p$ makes a decision, and let $v$ be $p$ 's decision value. Assume that process $q$ decides $v^{\prime}$ at phase $\phi$. By definition of $\phi_{0}$, we have $\phi \geq \phi_{0}$.

We proceed by contradiction, and assume that $v \neq$ $v^{\prime}$. Since $T>\frac{n}{2}+\alpha$, by Lemma 6 , we derive that $\phi>\phi_{0}$. Since $p$ decides $v$ at round $2 \phi_{0}$ and $q$ decides $v^{\prime}$ at round $2 \phi$, Lemma 5 ensures that $\left|Q^{2 \phi_{0}}(v)\right| \geq$ $T-\left|A H O\left(p, 2 \phi_{0}\right)\right|$ and $\left|Q^{2 \phi}\left(v^{\prime}\right)\right| \geq T-|A H O(q, 2 \phi)|$.

Since $T>\frac{2}{3}(n+2 \alpha)$, Lemma 8 implies that $Q^{2 \phi_{0}}(v)$ and $Q^{2 \phi}\left(v^{\prime}\right)$ are disjoint sets. Therefore,

$$
\left|Q^{2 \phi_{0}}(v) \cup Q^{2 \phi}\left(v^{\prime}\right)\right|=\left|Q^{2 \phi_{0}}(v)\right|+\left|Q^{2 \phi}\left(v^{\prime}\right)\right| .
$$

Because of $T>\frac{2}{3}(n+2 \alpha)$, we have $\mid Q^{2 \phi_{0}}(v) \cup$ $Q^{2 \phi}\left(v^{\prime}\right) \mid>n$, which is a contradiction.

Now we show that the $\mathrm{HO}$ machines $\left\langle B O T R, \mathcal{P}_{d y n}^{\alpha}\right\rangle$ satisfies the integrity clause of consensus for $T>2 \alpha$.

proposition 2 (Integrity). If $T>2 \alpha$, then in any run of the HO machine $\left\langle B O T R, \mathcal{P}_{d y n}^{\alpha}\right\rangle$ where all the initial values are equal to some value $v_{0}$, the only possible decision value is $v_{0}$.

Proof. Consider a run of the HO machine $\left\langle B O T R, \mathcal{P}_{d y n}^{\alpha}\right\rangle$ such that all the initial values are equal to $v_{0}$.

First, by induction on $r$, we show that:

$$
\forall r>0: Q^{r}\left(v_{0}\right)=\Pi
$$

Note that according to the code of BOTR, $p$ belongs to $Q^{r}\left(v_{0}\right)$ if and only if vote $_{p}^{(r-1)}=v_{0}$, and so $Q^{r}\left(v_{0}\right)=$ $\left\{p \in \Pi: \operatorname{vote}_{p}^{(r-1)}=v_{0}\right\}$.
Basic case: $r=1$. All the initial values are equal to $v_{0}$. Therefore, every process sends a message with value $v_{0}$ at round 1.

Inductive step: $r>1$. Suppose that $Q^{r-1}\left(v_{0}\right)=\Pi$. Let $p$ be a process that updates its variable $x_{p}$ at round $r-1$. Since $A H O(p, r-1) \leq \alpha$, each process $p$ receives at most $\alpha$ values distinct from $v_{0}$ at round $r-1$. Therefore, either $p$ does not modify vote $_{p}$ at the end of round $r$ which remains equal to $v_{0}$, or $p$ receives at least $T$ messages at round $r$, and thus at least $T-\alpha$ messages with value $v_{0}$ and at most $\alpha$ values different from $v_{0}$. In the latter case, $p$ sets vote $_{p}$ to $v_{0}$ since $T>2 \alpha$. This shows that definitely, vote ${ }_{p}^{(r-1)}=v_{0}$. Therefore, $Q^{r}\left(v_{0}\right)=\Pi$.

Let $p$ be a process that makes a decision at some round $r_{0}>0$. We have just shown that $Q^{r_{0}}\left(v_{0}\right)=\Pi$. When $\left|A H O\left(p, r_{0}\right)\right| \leq \alpha$ holds, $p$ receives at most $\alpha$ messages with value different to $v_{0}$. Since $T>\alpha$, the code entails $p$ to decide $v_{0}$ at round $r$.

For liveness, as already stated the communication predicate $\mathcal{P}_{\text {BOTR }}^{f}$ ensures that (i) vote $e_{q}$ are eventually identical, and (ii) each process then hears of sufficiently many processes to make a decision.

proposition 3 (Termination). If $n>4 \alpha+3 f$ and $T>\frac{2}{3}(n+2 \alpha)$, then any run of the HO machine $\left\langle\right.$ BOTR, $\left.\mathcal{P}_{\text {BOTR }}^{f} \wedge \mathcal{P}_{d y n}^{\alpha}\right\rangle$ satisfies the Termination clause of consensus.

Proof. Since $n>4 \alpha+3 f$, we have $n-f \geq T$. By $\mathcal{P}_{\text {BOTR }}^{f}$, there exists a phase $\phi_{0}$ such that for all processes $p\left|H O\left(p, 2 \phi_{0}-1\right)\right| \geq n-f \wedge \operatorname{CONS}\left(2 \phi_{0}-1\right)$. Therefore, in round $2 \phi_{0}-1$, for any two processes $p$ and $q$, we have $\vec{\mu}_{p}^{2 \phi_{0}-1}=\vec{\mu}_{q}^{2 \phi_{0}-1}$, and $\mid H O\left(p, 2 \phi_{0}-1\right) \cap H O\left(q, 2 \phi_{0}-\right.$ $1) \mid \geq n-f \geq T$. The code of BOTR algorithm (see line 8) implies that for all processes $p$ at the end of round $2 \phi_{0}-1$ we have vote $e_{p}$ set to the same value $v_{0}$.

Because of $T>\frac{2}{3}(n+2 \alpha)$, a similar argument as the one used in Lemma 8 shows that every process $q$ that updates vote $_{q}$ at round $r^{\prime}>r_{0}$ definitely sets it to $v_{0}$. Moreover, from $\mathcal{P}_{B O T R}^{f}$ we have $\forall p \in \Pi, \exists \phi_{1} \geq$ $\phi_{0}$ s.t. $\left|S H O\left(p, 2 \phi_{1}\right)\right| \geq n-f \geq T$. Therefore, there exist a round $2 \phi_{1}$ such that every process $p$ in $\Pi$ eventually receives at least $T$ messages with value $v_{0}$ at round $2 \phi_{1}$, and so decides $v_{0}$.

Combining Propositions 1, 2, and 3, we get the following theorem:

Theorem 2. If $n>4 \alpha+3 f$ and $T>\frac{2}{3}(n+2 \alpha)$, then the HO machine $\left\langle\right.$ BOTR, $\left.\mathcal{P}_{\text {BOTR }}^{f} \wedge \mathcal{P}_{\text {dyn }}^{\alpha}\right\rangle$ solves consensus.

Similar reasoning can be used to show: 


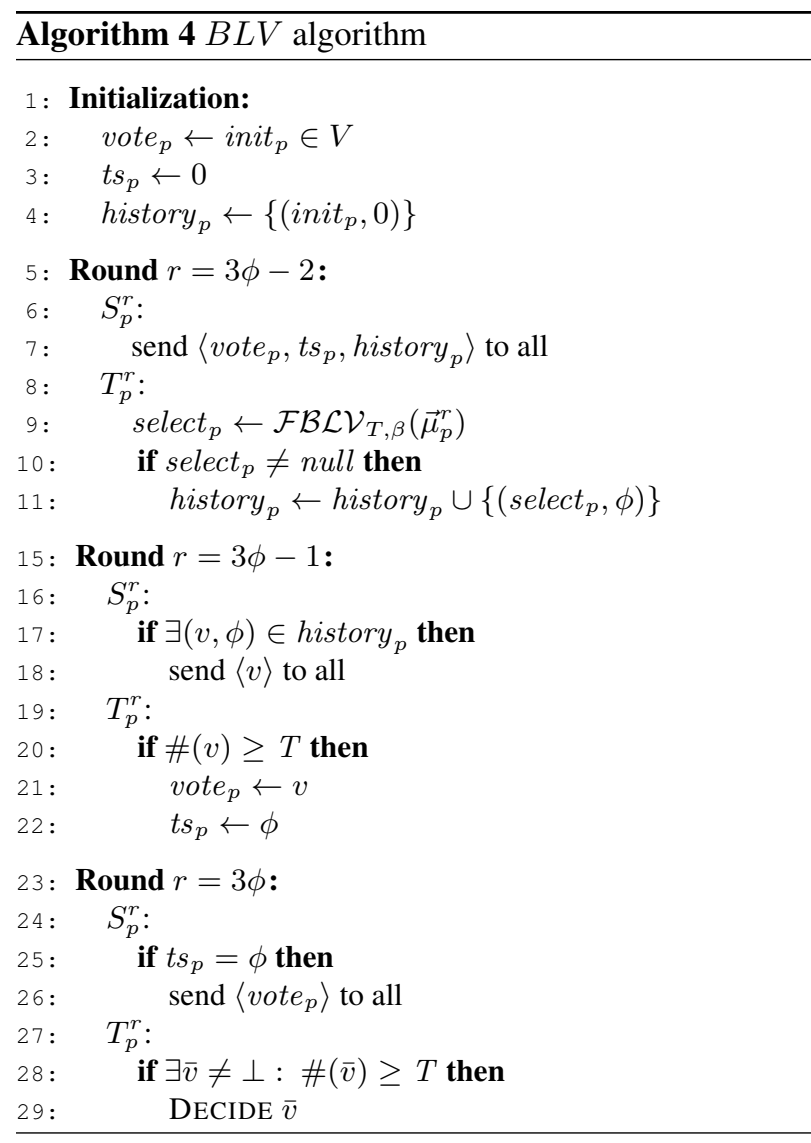

Corollary 6. If $\alpha=f, n>5 f$ and $T>\frac{2}{3}(n+f)$, then the HO machine $\left\langle B O T R, \mathcal{P}_{\text {BOTR }}^{f} \wedge \mathcal{P}_{\text {stat }}^{\alpha}\right\rangle$ solves consensus.

\subsection{The $B L V$ algorithm}

The next algorithm we present is called $B L V$. It is based on the last voting mechanism [11] that was first introduced in the seminal Paxos algorithm by Lamport [15] for benign faults. This mechanism is also at the core of the PBFT algorithm by Castro and Liskov [10], the Byzantine variant of the Paxos algorithm.

$B L V$ is designed to work both under $\mathcal{P}_{\text {stat }}^{\alpha}$ and $\mathcal{P}_{d y n}^{\alpha}$. It requires $n>2(\alpha+f)$ and $T>\frac{n}{2}+\alpha$ in the presence of dynamic value faults $\left(\mathcal{P}_{d y n}^{\alpha}\right)$, or $n>3 f, \alpha=f$, and $T>$ $\frac{n+f}{2}$ if value faults are only static $\left(\mathcal{P}_{\text {stat }}^{\alpha}\right)$. Termination is achieved with $\mathcal{P}_{d y n}^{\alpha}$ or $\mathcal{P}_{\text {stat }}^{\alpha}$ if in addition the following predicate holds:

$$
\begin{aligned}
\mathcal{P}_{B L V}^{f}: & : \forall \phi>0, \exists \phi_{0}>\phi: \operatorname{CONS}\left(3 \phi_{0}-2\right) \\
& \wedge \forall r \in\left\{3 \phi_{0}-2, \ldots, 3 \phi_{0}\right\}:|S K(r)| \geq n-f
\end{aligned}
$$

Obviously, $\mathcal{P}_{\diamond \text { cons } \oplus S K}^{f, 0,3}$ implies $\mathcal{P}_{B L V}^{f}$. Eventual consistency ensures that at the end of round $3 \phi_{0}-2$, all processes select the same value. The condition that there exists a large enough safe kernel in phase $\phi_{0}$ finally forces every process to make a decision at the end of round $3 \phi_{0}$.

The code of $B L V$ is given as Algorithm 4. It consists of a sequence of phases, where each phase $\phi$ has three rounds $3 \phi-2,3 \phi-1$ and $3 \phi$. The last voting mechanism uses a timestamp variable $t s$ in addition to to the variable vote. Whenever a process $p$ updates vote $_{p}$ in round $3 \phi-$ $1, t s_{p}$ is set to $\phi$ (line 21 and 22). If enough processes update vote in round $3 \phi-1$, then a decision is possible in phase $3 \phi$. This is the same mechanism as in Paxos. Note the condition at line 20. It ensures that in round $3 \phi-1$, all processes that update vote, update it to the same value. As in Paxos, this ensures that in round $3 \phi$, processes attempt to decide on one single value, which is necessary for agreement.

In order to deal with value faults, $B L V$ maintains also a history variable, which stores pairs $(v, \phi)$. Having $(v, \phi) \in$ history $_{p}$ means that $p$ added $(v, \phi)$ to history in phase $\phi$ (line 11). The history variable ensures that a corrupted message with invalid values for vote and $t s_{p}$ will not affect the safety properties of the algorithm. It is mainly used in round $3 \phi-2$, which has two roles, the first related to agreement and integrity, and the second related to termination:

1. Safety role:

(a) Agreement: If a process $p$ has decided $v$ in some phase $\phi_{0}$, then for any process $q$, only $v$ can be assigned to select $_{q}$ at line 9 in phases $\phi>\phi_{0}$.

(b) Integrity: If all process have the same initial value $v$, then only $v$ can be assigned to select $p$ at line 9 .

2. Termination role: In a consistent round with safe kernel of size $n-f$, all processes must assign the same value to select at line 9 .

Line 9 refers to the selection function $\mathcal{F B} \mathcal{B} \mathcal{V}_{T, \beta}$, which takes as input the messages received in round $3 \phi-2$. We explain now this function (Algorithm 5):

- Line 30 (together with line 31) ensures 1a. More precisely, it ensures selection of the most recent vote in the history of some process. This is basically the same mechanism as in Paxos, adapted to transmission value faults. Selecting the most recent vote among the set of majority processes can be expressed 


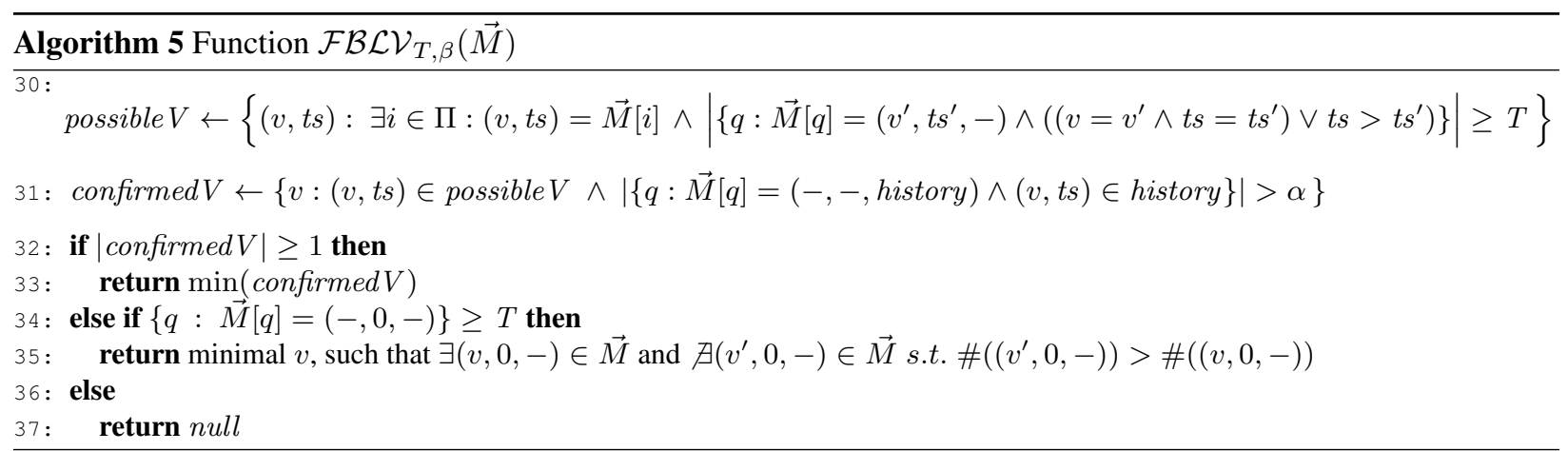

in Paxos as follows:

$$
\begin{array}{r}
\text { mostRecent } V \leftarrow\left\{(v, t s):(v, t s) \in \vec{\mu}_{p}^{r} \wedge\right. \\
\left.\left.\mid\left\{q: \vec{\mu}_{p}^{r}[q]=\left(v^{\prime}, t s^{\prime}\right) \wedge t s \geq t s^{\prime}\right)\right\} \mid>\frac{n}{2}\right\}
\end{array}
$$

In Paxos, this selection rule ensures agreement since most recent vote is a single value. In BLV, a corrupted message can contain (vote, $t s$ ) with $t s$ equal to the highest timestamp of a process, but with a different vote. Therefore, the above selection rule does not ensure 1a, since several values can satisfy the condition of lines 30 and 31 . The solution consists in transforming condition $t s \geq t s^{\prime}$ into $\left(v=v^{\prime} \wedge t s=t s^{\prime}\right) \vee t s>t s^{\prime}$ and using a higher threshold $\left(T>\frac{n}{2}+f\right.$ or $\left.T>\frac{n+f}{2}\right)$.

With this, if a process has previously decided $\bar{v}$, then only $\bar{v}$ can be in confirmed $V .^{11}$

- Line 31 prevents from returning a value $v$ from a pair $(v, t s)$ that is from a corrupted message: the pair must be in the history of at least one process. Therefore, a pair $(v, t s)$ is considered only if it is part of the history in at least $\alpha+1$ messages received. Together with line 30 , it also ensures $1 \mathrm{~b}$ : when all processes have the same initial value, no other value is in the history $_{p}$ variable of processes.

We consider now lines 32 and 33 of Algorithm 5. As we just explained, if a process has previously decided $\bar{v}$, then

\footnotetext{
${ }^{11}$ Consider two phases $\phi_{0}$ and $\phi_{0}+1$, such that a process has decided $\bar{v}$ in phase $\phi_{0}$. We consider the more general case in the presence of dynamic faults, and we assume that $n=5, f=\alpha=1$ and $T=4$. This means that at least $T-\alpha=3$ processes have $t s=\phi_{0}$ and vote $=\bar{v}$ Consider in phase $\phi_{0}+1$ that $(v, t s) \in \operatorname{possible}_{p}$ at $p$ with $v \neq \bar{v}$. This means that $p$, in round $3\left(\phi_{0}+1\right)-2$, has received $T=4$ messages with either $(v, t s,-)$, or $\left(-, t s^{\prime},-\right)$ and $t s^{\prime}<t s$. Since $n=5$ and $T=4$, at least one of these messages is from a process $c$ such that vote $_{c}=\bar{v}$ and $t s_{c}=\phi_{0}$. Since $v \neq \bar{v}$, we must have $\phi_{0}<t s$. However, in phase $\phi_{0}+1$, no process $p$ can have $(v, t s)$ with $t s>\phi_{0}$ in history $_{p}$. Therefore, by line 31 , we will not have $v \in$ confirmed $V$.
}

only $\bar{v}$ can be in confirmed $V$, that is, $\mid$ confirmed $V \mid=$ 1. In this case, by line 33 , the function $\mathcal{F B} \mathcal{B} \mathcal{V}_{T, \beta}$ returns $\bar{v}$. If no correct process has decided, we can have $\mid$ confirmed $V \mid>1$. In this case, if some round $3 \phi-2$ is a consistent round with safe kernel of size $n-f$, then all processes consider the same set confirmed $V$, which ensures 2. Lines 34 and 35 are for the case where not all processes have the same initial value. Termination would be violated without these lines.

\subsubsection{Correctness of the $B L V$ algorithm}

In this section we use the same definition of $R(v)$ and $Q(v)$ as in Section 6.1.1.

Definition 1. A value $v$ is locked in a phase $\phi$ by process $p$ if vote $_{p}=v$ and $t s_{p}=\phi$ at the end of round $3 \phi-1$.

Lemma 9. If $T>\frac{n}{2}+\alpha$, then in any run of the $\mathrm{HO}$ machine $\left(B L V, \mathcal{P}_{d y n}^{\alpha}\right)$ there is at most one locked value per phase.

Proof. Assume by contradiction that there exist two processes $p$ and $q$ that lock different values $v$ and $v^{\prime}$ in some phase $\phi_{0}>0$. From line 20 we deduce that $\left|R_{p}^{3 \phi_{0}}(v)\right| \geq T$ and $\left|R_{q}^{3 \phi_{0}}\left(v^{\prime}\right)\right| \geq T$. Then Lemma 5 (note that this lemma holds also for BLV) ensures that $\left|Q^{3 \phi_{0}}(v)\right| \geq T-\alpha$ and $\left|Q^{3 \phi_{0}}\left(v^{\prime}\right)\right| \geq T-\alpha$ when $\mathcal{P}_{d y n}^{\alpha}$ holds.

Since each process sends the same value to all at each round, the sets $Q^{3 \phi_{0}}(v)$ and $Q^{3 \phi_{0}}\left(v^{\prime}\right)$ are disjoint if $v$ and $v^{\prime}$ are distinct values. Hence,

$\left|Q^{3 \phi_{0}}(v) \cup Q^{3 \phi_{0}}\left(v^{\prime}\right)\right|=\left|Q^{3 \phi_{0}}(v)\right|+\left|Q^{3 \phi_{0}}\left(v^{\prime}\right)\right| \geq 2 T-2 \alpha$.

Consequently, since $T>\frac{n}{2}+\alpha$, we derive that $\left|Q^{3 \phi_{0}}(v) \cup Q^{3 \phi_{0}}\left(v^{\prime}\right)\right|>n$, a contradiction.

Lemma 10. If $T>\alpha$, then in any run of the HO machine $\left(B L V, \mathcal{P}_{d y n}^{\alpha}\right)$ there is at most one possible decision value per phase. 
Proof. Assume by contradiction that there exist two processes $p$ and $q$ that decide on different values $v$ and $v^{\prime}$ in some phase $\phi_{0}>0$. From line 28 we deduce that $\left|R_{p}^{3 \phi_{0}}(v)\right| \geq T$ and $\left|R_{q}^{3 \phi_{0}}\left(v^{\prime}\right)\right| \geq T$. Then Lemma 5 and $\mathcal{P}_{d y n}^{\alpha}$ ensure that $\left|Q^{3 \phi_{0}}(v)\right| \geq T-\alpha$ and $\left|Q^{3 \phi_{0}}\left(v^{\prime}\right)\right| \geq$ $T-\alpha$.

Since each process sends the same value to all at each round, the sets $Q^{3 \phi_{0}}(v)$ and $Q^{3 \phi_{0}}\left(v^{\prime}\right)$ are disjoint since $v$ and $v^{\prime}$ are distinct values. Hence, when $T>\alpha$, the sets $Q^{3 \phi_{0}}(v)$ and $Q^{3 \phi_{0}}\left(v^{\prime}\right)$ are not empty, and so by line 25, there exist two processes $p^{\prime}$ and $q^{\prime}$ that have vote $p_{p^{\prime}}=v$, $t s_{p^{\prime}}=\phi_{0}$, vote $q_{q^{\prime}}=v^{\prime}$ and $t s_{q^{\prime}}=\phi_{0}$. A contradiction with Lemma 9.

Lemma 11. If $T>\frac{n}{2}+\alpha$, then in any run of the $H O$ machine $\left(B L V, \mathcal{P}_{d y n}^{\alpha}\right)$, if process $p$ decides $v$ in phase $\phi_{0}>0$, then for all later phases $\phi>\phi_{0}$ and all processes $q,(v, \phi)$ is the only pair that can be added to history . $^{.}$

Proof. Assume by contradiction that $\phi_{1}>\phi_{0}$ is the first phase where a pair $\left(v_{1}, \phi_{1}\right)$ with $v_{1} \neq v$ is added to the history $_{q}$ at process $q$. This implies that if history at some process contains a pair $\left(v^{\prime}, \phi^{\prime}\right)$ with $v^{\prime} \neq v$, then $\phi^{\prime} \leq \phi_{0}$ (*).

Since by our assumption $q$ added $\left(v^{\prime},-\right)$ to history $_{q}$ in phase $\phi_{1}$, this implies that $\mathcal{F} \mathcal{B L} \mathcal{V}_{T, \beta}$ returns $v^{\prime}$ at line 9 in phase $\phi_{1}$. Therefore, either (i) line 33 or (ii) line 35 of Algorithm 5 was executed by $q$ in phase $\phi_{1}$.

In case (ii), the condition of line 34 has to be true. This implies that $\left|R_{q}^{3 \phi_{1}-2}((-, 0,-))\right| \geq T$, and thus, by Lemma 5, $\left|Q^{3 \phi_{1}-2}((-, 0,-))\right| \geq T-\alpha$.

We prove that in phases $\phi$ such that $\phi_{0} \leq$ $\phi<\phi_{1}$, we have $\mid\left\{q \in \Pi:\right.$ vote $_{q}^{3 \phi-1}=$ $\left.v \wedge t s_{q}^{3 \phi-1} \geq \phi_{0}\right\} \mid \geq T-\alpha$. Since $p$ decides $v$ in phase $\phi_{0},\left|R_{p}^{3 \phi_{0}}(v)\right| \geq T$, and thus by Lemma 5 , we have $\left|Q^{3 \phi_{0}}(v)\right| \geq T-\alpha$. From the code of the $B L V$ algorithm, we have $Q^{3 \phi_{0}}(v)=\left\{q: \operatorname{vote}_{q}^{3 \phi_{0}-1}=v \wedge t s_{q}^{3 \phi_{0}-1}=\right.$ $\left.\phi_{0}\right\}$, therefore the claim holds for phase $\phi_{0}$.

We now show that any process that locked value $v$ in phase $\phi_{0}$ (see Definition 1) and updates vote in phase $\phi$ such that $\phi_{0}<\phi<\phi_{1}$, sets it to $v$. This ensures the claim. Assume by contradiction that one of these processes $q^{\prime}$ sets vote $_{q}$ to $v^{\prime}$ in round $3 \phi-1$. By line 20 , $\left|R_{q}^{3 \phi-1}\left(v^{\prime}\right)\right| \geq T$. Then Lemma 5 ensures that $\left|Q^{3 \phi-1}\left(v^{\prime}\right)\right| \geq T-\alpha$. Since $T>\alpha$, we have $\left|Q^{3 \phi-1}\left(v^{\prime}\right)\right|>0$, i.e. at least one process sent $v^{\prime}$ at line 18 . Therefore, by line 17 at least one process has $\left(v^{\prime}, \phi_{0}+1\right)$ in history, a contradiction with the assumption that $\phi_{1}$ is the first phase where a pair $\left(v^{\prime},-\right)$ is added to history at some process.

So we have also $\left|\bigcup_{t s \geq \phi_{0}} Q^{3 \phi_{1}-2}((v, t s,-))\right| \geq T-$ $\alpha$. Since in each round, every process sends the same value to all, and $\phi_{0}>0$, the sets $X(v)=$ $\bigcup_{t s \geq \phi_{0}} Q^{3 \phi_{1}-2}((v, t s,-))$ and $Q^{3 \phi_{1}-2}((-, 0,-))$ are disjoint. Hence,

$$
\begin{aligned}
& \left|X(v) \cup Q^{3 \phi_{1}-2}((-, 0,-))\right|= \\
& |X(v)|+\left|Q^{3 \phi_{1}-2}((-, 0,-))\right| \geq 2 T-2 \alpha .
\end{aligned}
$$

Together with $T>\frac{n}{2}+\alpha$, we derive that $\mid \bigcup_{t s \geq \phi_{0}}$ $Q^{3 \phi_{1}-2}((v, t s,-)) \cup Q^{3 \phi_{1}-2}((-, 0,-)) \mid>n$, a contradiction.

In case (i), the condition at line 32 , has to be true, i.e., $v^{\prime}$ need to be part of confirmed $V$ set at line 31 . Value $v^{\prime}$ can be part of the set confirmed $V$ only if $\left(v^{\prime}, t s^{\prime}\right)$ is part of the set possible $V$ at line 30. We show that if $\left(v^{\prime}, t s^{\prime}\right)$ is part of the set possible $V$ at line $30, v^{\prime}$ cannot be part of the set confirmed $V$ at line 31, which establishes the contradiction.

If the pair $\left(v^{\prime}, t s^{\prime}\right)$ is added to the set possible $V$ at line 30 , then $H O\left(q, 3 \phi_{1}-2\right) \geq T$. Since $2 T-\alpha>$ $n+\alpha,\left|H O\left(q, 3 \phi_{1}-2\right) \cap \bigcup_{t s \geq \phi_{0}} Q^{3 \phi_{1}-2}((v, t s,-))\right|>\alpha$. Therefore, since $\mathcal{P}_{d y n}^{\alpha}$ holds, any set of messages of size $T$ contains at least one message $m$ with $m$.vote $=v$ and m.ts $\geq \phi_{0}(* *)$. So we have $\mid\left\{m^{\prime} \in \vec{\mu}_{q}^{r}:\left(m^{\prime} . t s<\right.\right.$ $\left.\left.t s^{\prime}\right)\right\} \mid \geq T$ and, because of $(* *), t s^{\prime}>\phi_{0}$.

The value $v^{\prime}$ is added to the set confirmed $V$ at line 31 only if there are at least $\alpha+1$ messages $\bar{m}$ in $\vec{\mu}_{q}^{3 \phi-2}$ such that: $(\bar{v}, \bar{\phi}) \in \bar{m}$.history and $\bar{\phi} \geq t s^{\prime}$ and $\bar{v}=v^{\prime}$. Since $t s^{\prime}>\phi_{0}$, by $(*), q$ receives at most $\alpha$ such messages, a contradiction.

proposition 4 (Agreement). If $T>\frac{n}{2}+\alpha$, then no two processes can decide differently in any run of the $\mathrm{HO}$ machine $\left(B L V, \mathcal{P}_{d y n}^{\alpha}\right)$.

Proof. Let a phase $\phi_{0}>0$ be the first phase at which some process $p$ makes a decision, and let $v$ be the $p$ 's decision value. Assume that process $q$ decides $v^{\prime}$ at phase $\phi^{\prime}$. By definition of $\phi_{0}$, we have $\phi^{\prime} \geq \phi_{0}$.

We proceed by contradiction and assume that $v \neq v^{\prime}$. By Lemma 10, we derive that $\phi^{\prime}>\phi_{0}$. Since $q$ decides at round $3 \phi^{\prime}$, by line 28 we have $\left|R_{q}^{3 \phi^{\prime}}\left(v^{\prime}\right)\right| \geq T$. By Lemma 5, we have $\left|Q^{3 \phi^{\prime}}\left(v^{\prime}\right)\right| \geq T-\alpha$. Since $T>\alpha$, there is at least one process $p^{\prime}$ that sends $v^{\prime}$ in round $3 \phi^{\prime}$. By line 25 and line 22 we have that process $p^{\prime}$ sends it's current vote in round $3 \phi^{\prime}$ only if vote is updated in round 
$3 \phi^{\prime}-1$. Therefore, $\left|R_{p^{\prime}}^{3 \phi^{\prime}-1}\left(v^{\prime}\right)\right| \geq T$, i.e. by Lemma 5 , we have $\left|Q^{3 \phi^{\prime}-1}\left(v^{\prime}\right)\right| \geq T-\alpha$. Since $T>\alpha$, at least one process $q^{\prime}$ sends $v^{\prime}$ in round $3 \phi^{\prime}-1$. By line 17, if $q^{\prime}$ sends $v^{\prime}$ in round $3 \phi^{\prime}-1$, then $\exists\left(v^{\prime}, \phi^{\prime}\right) \in$ history $q_{q^{\prime}}^{3 \phi^{\prime}-2}$, a contradiction with Lemma 11.

Lemma 12. If $T>2 \alpha$, then in any run of the HO machine $\left(B L V, \mathcal{P}_{d y n}^{\alpha}\right)$ where all the initial values are equal to some value $v$, for all processes $q$, history , contains $^{2}$ only pairs $(v,-)$.

Proof. Since all processes have $v$ as their initial value, history at all processes is initialized to $(v, 0)$. Assume by contradiction that $\phi_{0}$ is the first phase where a pair $\left(v^{\prime},-\right)$ is added to history $y_{p}$ at some process $p(*)$. This implies that $\mathcal{F} \mathcal{B} \mathcal{L} \mathcal{V}_{T, \beta}$ returns $v^{\prime}$ at line 9. Therefore, either (i) line 33 or (ii) line 35 of Algorithm 5 was executed by $p$ in phase $\phi_{0}$.

For (i), the condition at line 32 has to be true, i.e., $v^{\prime}$ needs to be in confirmed $V$ at line 31 . This means that $p$ received more than $\alpha$ messages $m=(-,-$, history $m)$ with $\left(v^{\prime}, t s\right) \in$ history $y_{m}$ in round $3 \phi_{0}-2$. By Lemma 5 and $\mathcal{P}_{d y n}^{\alpha}$, at least one process sends a message $m=$ $\left\langle-,-\right.$, history $\left.y_{m}\right\rangle$ with $\left(v^{\prime}, t s\right) \in$ history $_{m}$ in round $3 \phi_{0}-2$, a contradiction with (*).

For (ii), the condition of line 34 has to be true. If this condition is true, this implies that $\left|H O\left(p, 3 \phi_{0}-2\right)\right| \geq T$. Since $T>2 \alpha, \mathcal{P}_{d y n}^{\alpha}$ holds, and all processes have the same initial value $v, v$ is returned at line 35 and $\left(v, \phi_{0}\right)$ is added to the history $_{p}$. A contradiction.

proposition 5 (Integrity). If $T>2 \alpha$, then in any run of the HO machine $\left(B L V, \mathcal{P}_{d y n}^{\alpha}\right)$ where all the initial values are equal to some value $v$, the only possible decision value is $v$.

Proof. By contradiction, assume that phase $\phi_{0}>0$ is the first phase in which some process $p$ decides $v^{\prime} \neq v$.

Since $p$ decides at round $3 \phi_{0}$, by line 28 we have $\left|R_{p}^{3 \phi_{0}}\left(v^{\prime}\right)\right| \geq T$. By Lemma 5 and $\mathcal{P}_{d y n}^{\alpha}$, we have $\left|Q^{3 \phi_{0}}\left(v^{\prime}\right)\right| \geq T-\alpha$. Since $T>\alpha$, there is at least one process $q$ that sends $v^{\prime}$ in round $3 \phi_{0}$. By line 25 and line 22, we have that process $q$ sends it's current vote in round $3 \phi_{0}$ only if vote is updated in round $3 \phi_{0}-1$. Therefore, $\left|R_{q}^{3 \phi_{0}-1}\left(v^{\prime}\right)\right| \geq T$, i.e., by Lemma 5 and $\mathcal{P}_{d y n}^{\alpha}$, we have $\left|Q^{3 \phi_{0}-1}\left(v^{\prime}\right)\right| \geq T-\alpha$. Since $T>\alpha$, at least one process $q^{\prime}$ sends $v^{\prime}$ in round $3 \phi_{0}-1$. By line 17, if $q^{\prime}$ sends $v^{\prime}$ in round $3 \phi_{0}-1$, then $\exists\left(v^{\prime}, \phi_{0}\right) \in$ history $q_{q^{\prime}}^{3 \phi_{0}-2}$, a contradiction with Lemma 12.

proposition 6 (Termination). If $n>2(f+\alpha), T>$ $\frac{n}{2}+\alpha$ and $f \leq \alpha$, then any run of of the HO machine
$\left(B L V, \mathcal{P}_{d y n}^{\alpha} \wedge \mathcal{P}_{B L V}^{f}\right)$ satisfies the Termination clause of consensus.

Proof. By $\mathcal{P}_{B L V}^{f}$, there exists a phase $\phi_{0}$ such that

$\operatorname{CONS}\left(3 \phi_{0}-2\right) \wedge \forall r \in\left\{3 \phi_{0}-2, \ldots, 3 \phi_{0}\right\}: S K(r) \geq n-f$.

Therefore, in round $3 \phi_{0}-2$, for any two processes $p$ and $q$, we have $\vec{\mu}_{p}^{r}=\vec{\mu}_{q}^{r}$, and $\mid S H O\left(p, 3 \phi_{0}-2\right) \cap S H O\left(q, 3 \phi_{0}-\right.$ 2) $\mid \geq n-f$.

Part A. We now prove that select ${ }_{p}^{3 \phi_{0}-2}$ will be the same at all processes $p$, i.e., that $\mathcal{F} \mathcal{B} \mathcal{L} \mathcal{V}_{T, \beta}$ returns the same value at all processes, and all processes add the same pair to history in round $3 \phi_{0}-2$. There are two cases to consider: (i) some process $p \in S K\left(\phi_{0}\right)$ locked a value in some phase smaller than $\phi_{0}$, or (ii) there is no such process in $S K\left(\phi_{0}\right)$.

Case (i): Let $\phi<\phi_{0}$ be the largest phase in which some process $p$ locked some value $v$ (line 21). By Lemma 9 and since $Q>\frac{n}{2}+\alpha$, all processes that lock a value in phase $\phi$, lock the same value $v$. Since $n>2(f+\alpha)$ and $T>\frac{n}{2}+\alpha, n-f \geq T$; therefore in case (i) at least a pair $(v,-)$ is added to the set possible $V$ at line 30 of Algorithm $5(*)$.

We consider now line 31 of Algorithm 5. If $p$ locked value $v$ in phase $\phi$, then $\left|R_{p}^{3 \phi-1}(v)\right| \geq T$, i.e., by Lemma 5, we have $\left|Q^{3 \phi-1}(v)\right| \geq T-\alpha$ when $\mathcal{P}_{d y n}^{\alpha}$ holds. Because of line 17 of Algorithm 4, at least $T-\alpha$ processes have $(v, \phi)$ in history. By assumption, $n>$ $2(f+\alpha)$ and $T>\frac{n}{2}+\alpha$, therefore $n-f+T>n+\alpha$. Therefore, because of $\left|S K\left(\phi_{0}\right)\right| \geq n-f$, any set of messages received in round $3 \phi_{0}-2$ contains more than $\alpha$ messages $m$ with $(v, \phi) \in m$.history. Since $n>2(f+\alpha)$ and $T>\frac{n}{2}+\alpha, n-f \geq T$ (***), and therefore $v$ is added to the set confirmed $V$ at line 31 of Algorithm 5 $(* *)$.

From $(*)$ and $(* *)$, it follows that the condition of line 32 of Algorithm 5 is true at all processes in phase $\phi_{0}$. Moreover, since function $\mathcal{F} \mathcal{B L} \mathcal{V}_{T, \beta}$ is deterministic and $\operatorname{CONS}\left(3 \phi_{0}-2\right)$ holds, for any two processes $p$ and $q$, we have select $_{p}=$ select $_{q}$ at line 9 . Therefore $p$ and $q$ add the same pair to history at line 11 .

Case (ii): By hypothesis, for all processes $p \in S K\left(\phi_{0}\right)$, we have $t s_{p}=0$. By (***) $n-f \geq T$ and therefore the condition at line 34 of Algorithm 5 is true at each process. Moreover, by $\operatorname{CONS}\left(3 \phi_{0}-2\right)$ we have for any two processes $p$ and $q \vec{\mu}_{p}^{r}=\vec{\mu}_{q}^{r}$. Therefore, the value returned at line 35 of Algorithm 5 is the same at all processes, and they will add the same pair to history at line 11 of Algorithm 4.

Part B. From Part A, there exists a value $v$ such that at all processes $p$ we have $\left(v, \phi_{0}\right) \in$ history $_{p}$ at the beginning of round $3 \phi_{0}-1$. Therefore all processes send $v$ to 
all at line 18. By $\left|S K\left(3 \phi_{0}-1\right)\right| \geq n-f$ we have that all processes receive at least $n-f$ messages equal to $v$, and since by (***) $n-f \geq T$, they all set vote $p$ to $v$ (line 21 ) and send $v$ to all at line 26. By $\left|S K\left(3 \phi_{0}\right)\right| \geq n-f$ and the same reasoning we can show that all processes receive $n-f$ messages equal to $v$ in round $3 \phi_{0}$, and since by (***) $n-f \geq T$, decide $v$ at line 29 in phase $\phi_{0}$.

Combining Propositions 4, 5, and 6, we get the following theorem:

Theorem 3. If $n>2(\alpha+f)$ and $T>\frac{n}{2}+\alpha$, then the HO machine $\left\langle B L V, \mathcal{P}_{B L V}^{f} \wedge \mathcal{P}_{d y n}^{\alpha}\right\rangle$ solves consensus.

Similar reasoning can be used to show:

Theorem 4. If $\alpha=f, n>3 f$ and $T>\frac{n+f}{2}$, then the HO machine $\left\langle B L V, \mathcal{P}_{B L V}^{f} \wedge \mathcal{P}_{\text {stat }}^{\alpha}\right\rangle$ solves consensus.

\subsection{The $B L K$ algorithm}

The third algorithm we present is called $B L K$. It is based on locking/unlocking mechanism that was first introduced in the seminal consensus algorithm for benign and arbitrary faults given by Dwork, Lynch and Stockmayer [12].

It requires $n>2(\alpha+f)$ and $T>\frac{n}{2}+\alpha$ in the presence of dynamic value faults $\left(\mathcal{P}_{d y n}^{\alpha}\right)$, or $n>3 f, \alpha=f$ and $T>\frac{n+f}{2}$ if value faults are only static $\left(\mathcal{P}_{\text {stat }}^{\alpha}\right)$. The code of $B L K$ is given as Algorithm 6. It consists of a sequence of phases, where each phase $\phi$ has three rounds $3 \phi-2,3 \phi-1$, and $3 \phi$. In addition to the variable vote, and similarly to $B L V$, the algorithm maintains a timestamp ts and a history variable. In round $3 \phi-2$, every process $p$ sends $\left\langle\right.$ vote $_{p}$, init $\left._{p}\right\rangle$ to all, where init $_{p}$ is $p$ 's initial value. It is maybe surprising to see that also the initial value init $_{p}$ is sent in the first round. The initial value is used only when vote $_{p}=$ NONE, as can be seen in the selection function $\mathcal{F B} \mathcal{L} \mathcal{K}_{T}$ (Algorithm 7). A value selected in round $3 \phi-2$ (lines 9 and 15) is sent to all in round $3 \phi-1$. If in round $3 \phi-1$, a process $p$ receives at least $T$ messages equal to some value $v$, it sets $v^{2} e_{p}$ to $v$ and $t s_{p}$ to $\phi$ (lines 18 and 19). Then we say that process $p$ locked value $v$ in phase $\phi$. If vote $_{p}=$ NoNE then process $p$ has not locked any value. In round $3 \phi$, a process $p$ sends $\left\langle\right.$ vote $_{p}$, ts $_{p}$, history $\left._{p}\right\rangle$ to all processes. If some value $v$ is locked in phase $\phi$ by sufficiently high quorum of processes, then a decision is possible in phase $\phi$ (line 24).

A value can be unlocked by process $p$ in round $3 \phi$, if $p$ learns that some process $q$ locked different value in higher phase $\left(t s_{q}>t s_{p} \wedge\right.$ vote $_{q} \neq$ vote $\left._{p}\right)$. In addition to vote and $t s, B L K$ maintains the history variable, which stores pairs $(v, \phi)$. Having $(v, \phi) \in$ history $_{p}$ means that

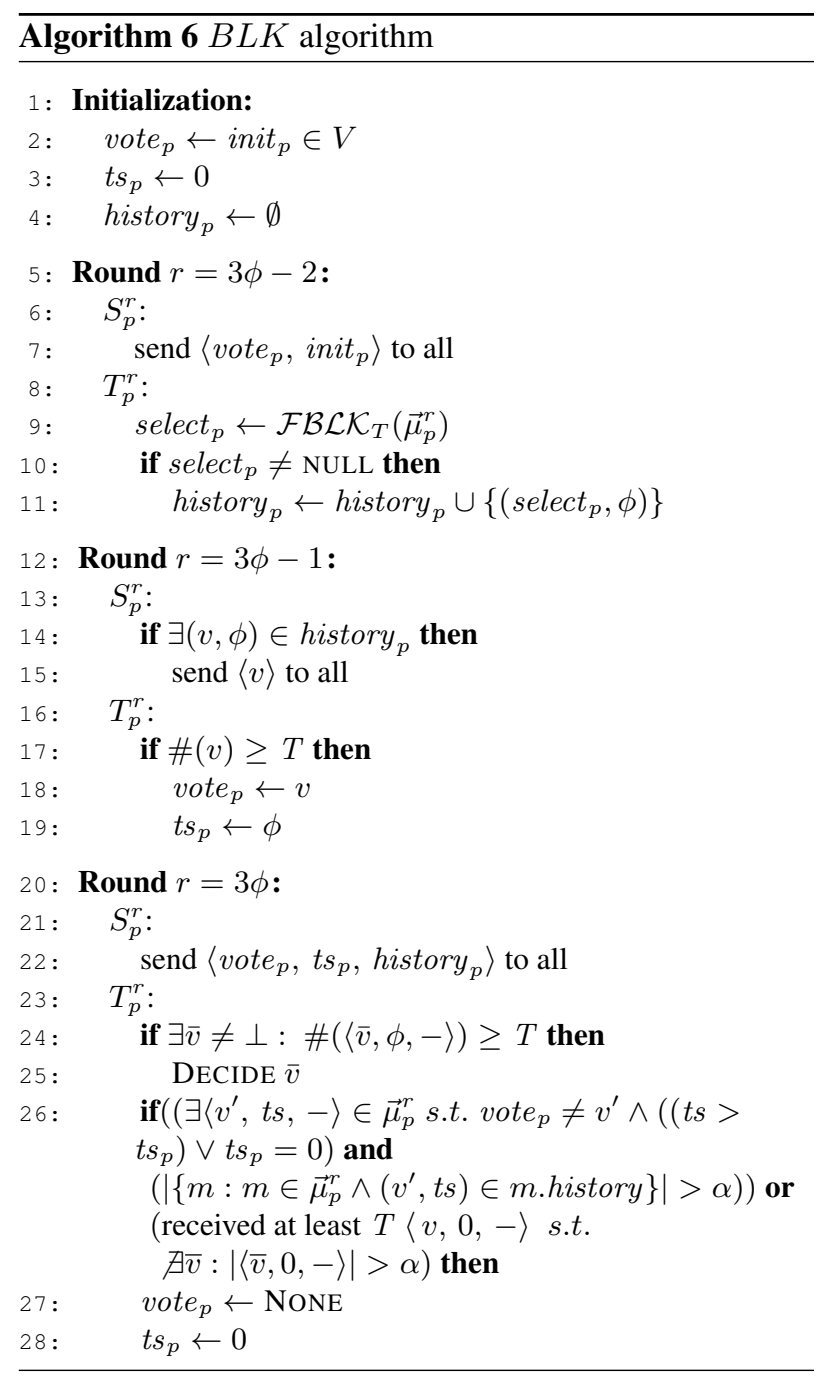

$p$ selected $v$ in round $3 \phi-2$ and added $(v, \phi)$ to history $_{p}$ in phase $\phi$ (line 11). It is used to filter out corrupted pairs (vote, ts) at round $3 \phi$.

It can be shown, using similar technique as for $B L V$, that $B L K$ is safe (it fulfills integrity and agreement) for appropriate choice of $T$ when $\mathcal{P}_{d y n}^{\alpha}$ holds (or $\mathcal{P}_{\text {stat }}^{\alpha}$, since $\mathcal{P}_{\text {stat }}^{\alpha}$ implies $\left.\mathcal{P}_{d y n}^{\alpha}\right)$. Termination is achieved in both cases if in addition the following predicate holds:

$$
\begin{aligned}
\mathcal{P}_{B L K}^{f}: & : \forall \phi>0, \exists \phi_{0}>\phi: \operatorname{CONS}\left(3 \phi_{0}-2\right) \\
& \wedge \forall r \in\left\{3 \phi_{0}-3, \ldots, 3 \phi_{0}\right\}:|S K(r)| \geq n-f
\end{aligned}
$$

Obviously, $\mathcal{P}_{\text {gen }}^{1,3}$ implies $\mathcal{P}_{B L K}^{f}$. Eventual consistency ensures that at the end of round $3 \phi_{0}-2$, all processes set select $_{p}$ to the same value. $\mathcal{P}_{B L K}^{f}$ also ensures a large enough safe kernel in the last round of the previous phase $\phi_{0}-1$. The role of this round is to ensure that all processes either lock the same value (those with the highest 


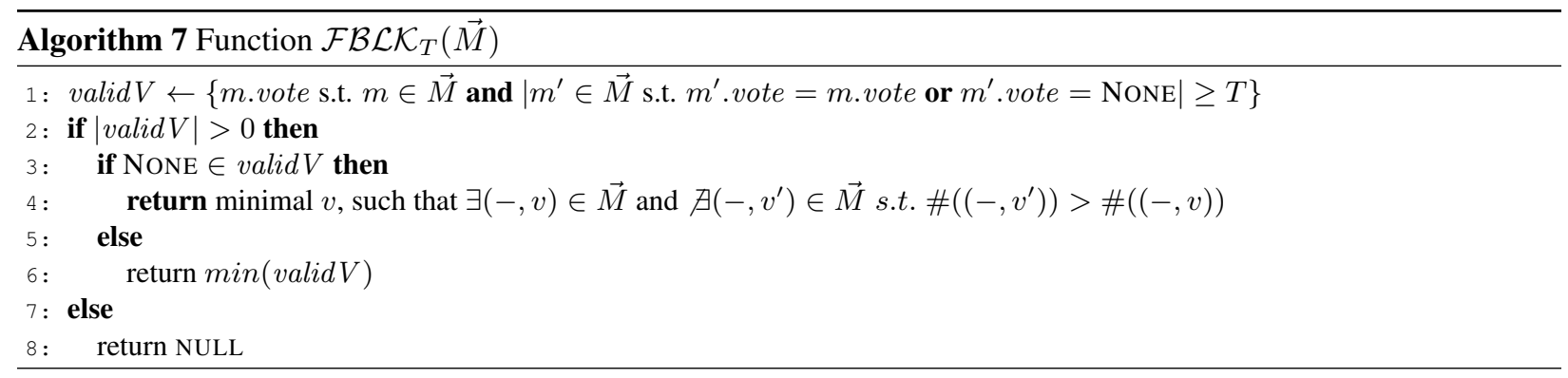

timestamp), or they do not lock any value. The condition that there exists a large enough safe kernel in phase $\phi_{0}$ finally forces every process to make a decision at the end of round $3 \phi_{0}$.

The proof of correctness follows a similar pattern as for $B L V$ and is not repeated here.

BLK versus BLV. There are strong similarities between BLV and BLK: three rounds per phase, only round $3 \phi-2$ must eventually be a consistent round, the history variable. However, the mechanisms for agreement differ: BLV uses a last voting mechanism, while BLK employs a locking mechanism. The two mechanisms are used in round $3 \phi-2$, when assigning a value to select (line 9):

- The last voting mechanism uses vote and ts (mechanism of PBFT and Paxos).

- The locking mechanism uses only vote (mechanism introduced in [12]).

This difference has consequences in the information sent in round $3 \phi-2$ : in BLV, $\left\langle\right.$ vote $_{p}$, ts $_{p}$, history $\left._{p}\right\rangle$ is sent; in BLK, only $\left\langle\right.$ vote $_{p}$, init $\left._{p}\right\rangle$ is sent. The initial value is only needed when several correct processes do not have a locked value (vote $=$ NONE) as can be seen in Algorithm 7 (see line 3 and 4).

To illustrate the difference between the two mechanisms, consider the case with dynamic value faults where $n=5, \alpha=f=1, T=4$ and some process $p_{1}$ has decided $v_{1}$ at the end of phase $\phi_{1}$. A possible configuration of processes $p_{1}$ to $p_{5}$ for the two algorithms at the end of phase $\phi_{1}$ is the following:

$$
\left(v_{1}, \phi_{1}\right),\left(v_{1}, \phi_{1}\right),\left(v_{1}, \phi_{1}\right)\left(v_{2}, \phi_{2}\right),\left(v_{2}, \phi_{2}\right)
$$

where each tuple represents the states (vote, $t s$ ) and $\phi_{2}<$ $\phi_{1} .{ }^{12}$ The history at $T-\alpha=3$ processes contains the pair $\left(v_{1}, \phi_{1}\right)$. In round $3\left(\phi_{1}+1\right)-2$ of the BLV algorithm, let

\footnotetext{
${ }^{12}$ Process $p_{1}$ decided $v_{1}$ by receiving correctly messages from processes $p_{1}, p_{2}$ and $p_{3}$ and the corrupted message $\left\langle v_{1}, \phi_{1},-\right\rangle$ from $p_{4}$.
}

a process $p_{2}$ receive, from processes $p_{1}$ to $p_{5}$ (the message received from process $p_{5}$ is corrupted):

$\left(v_{1}, \phi_{1},-\right),\left(v_{1}, \phi_{1},-\right),\left(v_{1}, \phi_{1},-\right),\left(v_{2}, \phi_{2},-\right),\left(v_{2}, \phi_{1},-\right)$.

With the last voting mechanism, we have $v_{1} \in$ confirmed $V$ (there are 4 messages with vote $=v_{1}$ or ts $<\phi_{1}$ and $\left(v_{1}, \phi_{1}\right)$ is in history of the message sent by three processes), and select $p_{p}$ is set to $v_{1}$. Assume that similarly, in round $3\left(\phi_{1}+1\right)-2$ of the BLKalgorithm, process $p_{2}$ receives, from processes $p_{1}$ to $p_{5}$ (all messages are correctly received):

$$
\left(v_{1},-\right),\left(v_{1},-\right),\left(v_{1},-\right),\left(v_{2},-\right),\left(v_{2},-\right) .
$$

With the locking mechanism, valid $V$ in Algorithm 7 is empty (there are no four messages with vote $=v_{1}$ ), and NULL is returned. With the locking mechanism, processes $p_{1}, p_{2}$ and $p_{3}$ have "locked" $v_{1}$, while processes $p_{4}$ and $p_{5}$ has "locked" $v_{2}$. It is clear that as long as processes $p_{4}$ and $p_{5}$ have locked $v_{2}$, no additional process can decide. Therefore, an unlocking mechanism is needed. This is the role of lines 26 and 28 of Algorithm 6. If process $p_{4}$ receives a message $(v, t s,-)$ from a process with $(v, t s)$ in history of $\alpha+1$ messages received, and vote $_{p_{4}} \neq v$, $t s_{p_{4}}<t s$, then process $p_{4}$ unlocks vote $_{p_{4}}$ by setting the variable to NONE (line 28). The second part of condition at line 26 is for the case where not all processes have the same initial value (termination would be violated without it). Now in round $3\left(\phi_{1}+1\right)-2$, let a process receive, from processes $p_{1}$ to $p_{5}$ :

$$
\left(v_{1},-\right),\left(v_{1},-\right),\left(v_{1},-\right),(\text { NONE },-),\left(v_{2},-\right) .
$$

This leads to have $v_{1} \in$ valid $V$, and select $p$ is set to $v_{1}$.

Observe that the unlocking mechanism requires history $_{p}$ (line 22). Therefore, we can also summarize the two mechanisms by saying that the last voting mechanism requires history $y_{p}$ in phase $3 \phi-2$, while the locking mechanism requires history in phase $3 \phi$ (for unlocking). 


\section{Deriving the overall resilience of $B L V$}

In this section we look at the overall resilience of the $B L V$ consensus algorithm together with the $\mathcal{P}_{B L V}^{f}$ predicate simulation algorithm. A similar derivation can be done for the BOTR and $B L K$ algorithms.

When solving consensus in the presence of (only) static value faults $\left(\mathcal{P}_{\diamond S K} \wedge \mathcal{P}_{\text {stat }}^{f}\right)$, both algorithms $(B L V$ and the simulation algorithm) require $n>3 f$. This follows from Theorem 4, Corollary 4 and the fact that $\mathcal{P}_{\diamond \text { cons } \oplus S K}^{f, 0,3}$ implies $\mathcal{P}_{B L V}^{f}$. However, these algorithms have different requirements on $n$ in the presence of dynamic value faults $\left(\mathcal{P}_{\diamond S K} \wedge \mathcal{P}_{d y n}^{f}\right)$.

From Corollary 5 and the fact that $\mathcal{P}_{\diamond \text { cons } \oplus S K}^{f, 0,3}$ implies $\mathcal{P}_{B L V}^{f}$ we get:

Corollary 7. If $n>\frac{(\beta+1)(\alpha+f)}{\beta-\alpha+1}, n>\alpha+f, \alpha \geq f$, and $\beta \geq \alpha$, then Algorithm 2 simulates $\mathcal{P}_{B L V}^{f} \wedge \mathcal{P}_{\text {dyn }}^{\beta}$ from $\mathcal{P}_{\diamond S K}^{6(f+1)+5} \wedge \mathcal{P}_{d y n}^{\alpha}$.

From Corollary 7 for any $\beta \geq \alpha$, we can simulate $\mathcal{P}_{B L V}^{f} \wedge \mathcal{P}_{d y n}^{\beta}$ from $\mathcal{P}_{\diamond S K}^{f, 6(f+1)+5} \wedge \mathcal{P}_{d y n}^{\alpha}$ if

$$
n>\frac{(\beta+1)(\alpha+f)}{\beta-\alpha+1} \wedge n>\alpha+f
$$

On the other hand, from Theorem 3 we know that we can solve consensus with BLV under $\mathcal{P}_{B L V}^{f} \wedge \mathcal{P}_{d y n}^{\beta}$ if

$$
n>2(\beta+f) .
$$

Combining these conditions and setting $\beta=k \alpha$, where $k \in \mathbb{R}, k \geq 1$, we can solve consensus with Algorithm 4 and Algorithm 2 under $\mathcal{P}_{\diamond S K}^{f, 6(f+1)+5} \wedge \mathcal{P}_{d y n}^{\alpha}$ if the following two conditions hold:

$$
\begin{aligned}
& n>\frac{(k \alpha+1)(\alpha+f)}{k \alpha-\alpha+1} \\
& n>2(k-1) \alpha+2(\alpha+f) .
\end{aligned}
$$

We first consider $\alpha>1$, then $\alpha=1$.

Case $\alpha>1$ : We can obtain different resilience bounds depending on the choice of $k$.

Choosing $k=1$ leads to the quadratic dependency from Corollary 2, and is thus not what we want to achieve here.

For $k \geq 2$, condition (3) implies condition (2) for any $\alpha>1$, because $\frac{k \alpha+1}{k \alpha-\alpha+1} \leq 2$. Thus, when choosing $k \geq$ 2 , the smallest $n$ is obtained with $k=2$ :

$$
n>4 \alpha+2 f .
$$

In case $1<k<2$, the optimal choice of $k$ depends on $\alpha$ and $f$. As special case we get for $k=1.5$ from condition (2), $n>\frac{3 \alpha+2}{\alpha+2}(\alpha+f)$, i.e.,

$$
n>3(\alpha+f)
$$

while from condition (3) we get

$$
n>3 \alpha+2 f
$$

Since both conditions should hold, it follows that $n>$ $3(\alpha+f)$.

Case $\alpha=1$ : For the special case $\alpha=1$ and $f=1$, conditions (3) and (2) become $n>2(k-1)+4$ and $n>$ $\frac{2(k+1)}{k}$. We obtain the smallest value for $n$ by choosing $k=1$, which leads to $n>4$.

Discussion: The results show that $k=1$ (i.e. $\beta=\alpha$ ) leads to the smallest value of $n$ only when $\alpha=1$. In cases where $\alpha>1$, a better choice is e.g. $k=1.5$ (i.e. $\beta=1.5 \alpha$ ). This is a non intuitive result.

\section{Direct implementation of eventual consistency using authentication}

In Section 5 we gave two simulations of $\mathcal{P}_{\diamond \text { cons }}$ from $\mathcal{P}_{\diamond S K}$. In this section we show that in some systems we can get $\mathcal{P}_{\diamond c o n s}$ with sufficiently high coverage without such a simulation, but simply using authentication. Authentication has been introduced very early in distributed computing research to solve consensus. Nevertheless, people were always struggling to give a rigorous formal definition of authentication.

The first observation is that in a transmission fault model, the introduction of authentication makes the model in fact benign: if every process signs its messages and upon reception only correctly signed messages are processed, no corruptions can occur. This implies that with authentication (whatever it means) transmission faults are not able to capture Byzantine process faults. However, even if we consider process faults, it is hard to formalize authentication in a precise manner. A possible approach to this open question is, instead of trying define authentication, state what can be achieved with authentication. As we will show, (eventual) consistency is what we naturally get from authentication assuming (eventual) synchrony. 


\subsection{Ensuring $\mathcal{P}_{I C}$ from synchrony and cor- rect leader using authentication}

Consistency, namely $\mathcal{P}_{I C}^{f}$ (Sect. 4.4), can be achieved with high probability using cryptographic signatures in a synchronous system with $f$ Byzantine processes (note that we are then no more in the scope of the transmission fault model; for a discussion for the relation between these two models see Section 9). To that end, in every round that should be consistent, every process signs its messages before sending it to the (correct) leader. The leader collects all the messages it receives and forwards them to all processes. The processes deliver all correctly signed messages that are received from the leader as the messages of this round. Technically this procedure requires two "subrounds" that can be obtained in a similar way as the normal round structure. However, the algorithm is not a simulation as in the previous section, since the correctness is conditional.

Assuming the (i) signatures cannot be forged, (ii) the system is synchronous and (iii) the leader is correct, it is easy to see that (a) all processes have the same reception vector, and (b) all processes receive at least $n-f$ messages. Therefore, $\mathcal{P}_{I C}^{f}$ holds.

\subsection{Ensuring $\mathcal{P}_{\diamond c o n s}$ from eventual syn- chrony and eventual correct leader us- ing authentication}

The above leader-based procedure can be used, with a small modification, to ensure $\mathcal{P}_{\diamond \text { cons }}$ from eventual synchrony. It is sufficient to replace the fixed correct leader with a rotating leader. This ensures an eventual correct leader when synchrony holds. The result follows directly.

\section{Communication predicates and corresponding systems}

In the HO model, there are no faulty processes and no state corruption. Nevertheless, for predicates that characterize permanent faults, the model can be used to reason about classical Byzantine faults. This implies that the algorithms in this paper can be used also to solve consensus in the classical Byzantine fault model. We develop this observation first for a synchronous system (for simplicity), and then extend it to our model. ${ }^{13}$

Let $S_{f}$ denote a synchronous system with reliable links and at most $f$ Byzantine processes, and consider on the

\footnotetext{
${ }^{13}$ This observation was made already in [19] and [5], but without giving algorithms supporting the observation.
}

other hand an HO machine with $|S K| \geq n-f$. For correct processes, a run in $S_{f}$ is indistinguishable from a run of the HO machine. Therefore, an algorithm that solves consensus with $|S K| \geq n-f$ allows in $S_{f}$ correct processes to solve consensus. Note that in $S_{f}$ faulty processes do not follow the protocol. It is then natural that they do not follow the specification of consensus.

The same indistinguishability argument can be applied to (i) the weaker partial synchronous system [12] with at most $f$ Byzantine processes and (ii) the $\mathrm{HO}$ model with $\mathcal{P}_{\text {stat }}^{f} \wedge \mathcal{P}_{\diamond S K}^{f, \infty}$. For correct processes in the model (i), a run is indistinguishable from a run in model (ii), and so an $\mathrm{HO}$ algorithm that solves consensus allows correct processes in the fault-prone system to solve consensus.

The predicate $\mathcal{P}_{d y n}^{\alpha} \wedge \mathcal{P}_{\diamond S K}^{f, k}, \alpha \geq f$, can correspond to a partially synchronous system with at most $f$ Byzantine processes, where in addition, before stabilization time, in every round processes can receive $\alpha-f$ corrupted messages from correct processes. This spectrum of interpretations, which includes permanent faults (see Sect. 4.3) contrary to [5], shows the benefit of considering the consensus problem in a model with (only) transmission faults.

\section{Related work}

Most research on consensus algorithms under arbitrary faults is considering component fault models, where faults are attached to a component that is either a process or a link. Furthermore, faults are mainly permanent: if a process or link commits a fault, the process/link is considered to be faulty during whole execution. It follows that not all components can be faulty, which is referred to as static faults. This explains that most research on consensus under arbitrary faults is about tolerating permanent and static process and/or link faults. Moreover, most of the literature considers only process faults, e.g., the classical Byzantine fault model where at most $f$ processes can behave arbitrarily. We can cite the early work of Lamport, Shostak and Pease [23, 18], which considers a synchronous system and gives algorithms for Interactive Consistency and Byzantine agreement together with matching lower bounds. Consensus in the partially synchronous model with Byzantine faults is considered in $[12,2,21,26]$. Byzantine variants of Paxos [15] include [10, 19, 1, 20, 17].

A few authors solve consensus in the synchronous system model where, in addition to Byzantine processes, a small number of links connecting correct processes may be arbitrary faulty during the entire execution of a consensus algorithm [25, 29, 33]. However, only a very limited number of links can be faulty. 
An alternative approach to component fault models is the transmission fault model that considers faults without trying to identify their cause. Pioneer work was done by Santoro and Widmayer in [27], with the introduction of transmission faults in synchronous systems. The transmission fault model is well-adapted to dynamic and transient faults. Santoro and Widmayer show in [27] that consensus cannot be solved if in every round as little as $\lfloor n / 2\rfloor$ messages are corrupted. The problem comes from the dynamic nature of faults, which can affect different processes in every round. The particularly problematic case is when these faults occur in blocks, i.e., in every round the outgoing links of one process are affected. Schmid et al. [30] give a lower bounds on link failures. They show the necessity of $n>4 f_{\ell}$, where $f_{\ell}$ is the maximum number of transmission faults that occur per sender and per receiver. In our model this can be expressed as

$$
\begin{aligned}
& \forall p \in \Pi, \forall r>0:|S H O(p, r)| \geq n-f_{\ell} \wedge \\
&|S T T(p, r)| \geq n-f_{\ell},
\end{aligned}
$$

where $\operatorname{STT}(p, r)$ represents safe talked-to sets [11], defined as $S T T(p, r)=\left\{q \in \Pi: \vec{\mu}_{q}^{r}[p]=S_{p}^{r}\left(s_{p}\right)\right\}$.

One approach to circumvent the impossibility result of Santoro and Widmayer is to restrict, per round, the number of transmission faults that correct processes may experience (both for outgoing and incoming messages) in order to avoid block of faults. This approach is considered in $[32,4,6]$ to solve consensus in the synchronous system model. The model considered is the hybrid fault model for synchronous systems, which extends the process fault model with communication failures. In this model, every process is allowed to commit up to $f_{l}^{s a}$ arbitrary send link failures and experience up to $f_{l}^{r a}$ arbitrary receive link failures without being considered as arbitrary faulty. This synchronous system model corresponds to a strong communication predicate where a large number of messages must be transmitted correctly in every round. Furthermore, tolerating additional $f_{s}$ send and $f_{r}$ receive omissions (i.e., message loss) requires to increase the number of processes by small multiples of $f_{s}$ and $f_{r}$.

An alternative approach to circumvent the impossibility results of $[27,30]$ is to distinguish between the safety and liveness conditions, as done by Biely et al. [5]. There, consensus under transmission faults (both benign and value faults) is solved the first time in a non-synchronous setting. For safety, only the number of corrupted messages is restricted, that is, in each round $r$ of the round based model, every process $p$ receives at most $\alpha$ corrupted messages. However, for liveness [5] assumes that there is a sufficiently large set $\Pi_{0}$ so that eventually there is a round $r$ where $\operatorname{SHO}(p, r)=H O(p, r)=\Pi_{0}$, for all processes $p$ in a sufficiently large set $\Pi_{1}$. This means that processes from a sufficiently large set $\Pi_{1}$ cannot receive even a single corrupted message in round $r$. Therefore, although the algorithms presented in [5] solve consensus under dynamic value faults for the first time in non-synchronous settings, they cannot tolerate permanent value faults located at a process, i.e., these algorithms do not solve consensus in systems with arbitrary process faults.

Our algorithms can also be used in the model considered in [5]. Note that for $f=0$, the predicate $\mathcal{P}_{B L V}^{f}$ satisfies the above liveness condition $(S H O(p, r)=$ $H O(p, r)=\Pi$ for all processes $p \in \Pi)$. Therefore, our algorithm $B L V$ solves consensus in the model of [5] if $n>2 \alpha$ (see Theorem 3, Section 6.2.1), in contrast to algorithm $\mathcal{A}_{T, E}$ in [5], which requires $n>4 \alpha$. Algorithm $\mathcal{U}_{T, E, \alpha}$ in [5] requires $n>2 \alpha$ but, contrary to $B L V$, requires for safety a permanent condition on liveness of communication: in every round every process receives sufficient number of correct messages. The algorithms presented in this paper are still correct even if processes does not receive any correct message in some rounds.

To summarize, the consensus algorithms presented in this paper avoid the drawbacks of previous approaches:

- By considering the transmission fault model, the algorithms can tolerate dynamic and transient faults in addition to only permanent and static faults of the component failure model. More precisely, the algorithms can tolerate arbitrary process faults and arbitrary link faults, where all links can be corrupted at some point during the system lifetime (contrary to only a few links in the algorithms designed for the component fault models).

- Contrary to most of the related work, our consensus algorithms can also be used in the partially synchronous system model and not just in the synchronous system model, weakening therefore the conditions on liveness of communication.

- Contrary to the algorithms in [5], our algorithms can also be used in systems with arbitrary faulty processes, which can potentially send corrupted messages in every round.

Finally, despite the similarity in title, [3] addresses a different topic. The paper investigates the possibility of designing protocols that are both self-stabilizing and faulttolerant in an asynchronous system. 


\section{Conclusion}

The transmission fault model allows us to reason about permanent and transient value faults in a uniform way, which makes the model very attractive. However, all existing solutions to consensus in this model are either in the synchronous system, or require strong conditions for termination that exclude the case where all messages of a process can be corrupted. The paper has shown that this limitation can be overcome thanks to the eventual consistency predicate that states the existence of a round where all processes receive the same set of messages. Two simulations of eventual consistency have been given, both from a predicate that corresponds to a partially synchronous system parameterized with $\alpha$ (in every round each process can receive up to $\alpha$ corrupted messages) and $f$ (at most $f$ processes are corrupted). The first simulation, which refers only to the parameter $f$, is for static faults. The second simulation, which refers to the parameters $f$ and $\alpha$, includes static and dynamic faults, and is compatible with permanent and transient faults. The paper has pointed out two options for this second simulation: preserving or not the number of corrupted messages in each round. The first option requires $n>(\alpha+1)(\alpha+f)$. The second option requires $n>\eta(\alpha+f)$. Combining the $B L V$ consensus algorithm with this second simulation leads to $n>3(\alpha+f)$ for $\alpha>1$ and $n>4$ for $\alpha=1$.

\section{References}

[1] I. Abraham, G. Chockler, I. Keidar, and D. Malkhi. Byzantine disk paxos: optimal resilience with byzantine shared memory. Distributed Computing, 18(5):387-408, 2006.

[2] M. K. Aguilera, C. Delporte-Gallet, H. Fauconnier, and $\mathrm{S}$. Toueg. Consensus with byzantine failures and little system synchrony. In Dependable Systems and Networks (DSN 2006), pages 147-155, 2006.

[3] E. Anagnostou and V. Hadzilacos. Tolerating transient and permanent failures (extended abstract). In Proceedings of the 7th International Workshop on Distributed Algorithms, WDAG '93, pages 174188. Springer-Verlag, 1993.

[4] M. Biely. An optimal Byzantine agreement algorithm with arbitrary node and link failures. In Proc. 15th Annual IASTED International Conference on Parallel and Distributed Computing and Systems (PDCS'03), pages 146-151, Marina Del Rey, California, USA, Nov. 3-5, 2003.
[5] M. Biely, B. Charron-Bost, A. Gaillard, M. Hutle, A. Schiper, and J. Widder. Tolerating corrupted communication. In Proceedings of the 26th Annual ACM Symposium on Principles of Distributed Computing (PODC'07). ACM Press, 2007.

[6] M. Biely, U. Schmid, and B. Weiss. Synchronous consensus under hybrid process and link failures. Theoretical Computer Science, In Press, Corrected Proof:-, 2010.

[7] F. Borran, M. Hutle, and A. Schiper. Timing analysis of leader-based and decentralized byzantine consensus algorithms. In $L A D C$, pages 166-175, 2011.

[8] F. Borran and A. Schiper. A leader-free byzantine consensus algorithm. In ICDCN, pages 67-78, 2010.

[9] F. V. Brasileiro, F. Greve, A. Mostéfaoui, and M. Raynal. Consensus in one communication step. In Proceedings of the 6th International Conference on Parallel Computing Technologies, PaCT '01, pages 42-50, London, UK, 2001. Springer-Verlag.

[10] M. Castro and B. Liskov. Practical byzantine fault tolerance and proactive recovery. ACM Transactions on Computer Systems, 20(4):398-461, 2002.

[11] B. Charron-Bost and A. Schiper. The heard-of model: computing in distributed systems with benign faults. Distributed Computing, 22(1):49-71, 2009.

[12] C. Dwork, N. Lynch, and L. Stockmeyer. Consensus in the presence of partial synchrony. Journal of the ACM, 35(2):288-323, Apr. 1988.

[13] M. J. Fischer, N. A. Lynch, and M. S. Paterson. Impossibility of distributed consensus with one faulty process. Journal of the ACM, 32(2):374-382, Apr. 1985.

[14] E. Gafni. Round-by-round fault detectors (extended abstract): unifying synchrony and asynchrony. In Proceeding of the 16th Annual ACM Symposium on Principles of Distributed Computing (PODC'98), pages 143-152, Puerto Vallarta, Mexico, 1998. ACM Press.

[15] L. Lamport. The part-time parliament. ACM Trans. Comput. Syst., 16:133-169, May 1998.

[16] L. Lamport. Fast paxos. Technical Report MSR-TR2005-12, Microsoft Research, 2005. 
[17] L. Lamport. Byzantizing paxos by refinement. In Proceedings of the 25th international conference on Distributed computing, DISC'11, pages 211-224, Berlin, Heidelberg, 2011. Springer-Verlag.

[18] L. Lamport, R. Shostak, and M. Pease. The byzantine generals problem. ACM Trans. Program. Lang. Syst., 4(3):382-401, 1982.

[19] B. Lampson. The abcd's of paxos. In Proceeding of the 19th Annual ACM Symposium on Principles of Distributed Computing (PODC'01), page 13. ACM Press, 2001.

[20] J.-P. Martin and L. Alvisi. Fast byzantine consensus. Transactions on Dependable and Secure Computing, 3(3):202-214, 2006.

[21] Z. Milosevic, M. Hutle, and A. Schiper. Unifying Byzantine consensus algorithms with weak interactive consistency. In 12th International Conference on Principles of Distributed Systems (OPODIS 2009), 2009.

[22] G. Neiger and S. Toueg. Automatically increasing the fault-tolerance of distributed algorithms. J. Algorithms, 11:374-419, September 1990.

[23] M. Pease, R. Shostak, and L. Lamport. Reaching agreement in the presence of faults. Journal of the ACM, 27(2):228-234, 1980.

[24] F. Pedone, A. Schiper, P. Urbán, and D. Cavin. Solving agreement problems with weak ordering oracles. In Proceedings of the 4th European Dependable Computing Conference on Dependable Computing, EDCC-4, pages 44-61, London, UK, 2002. Springer-Verlag.

[25] S. S. Pinter and I. Shinahr. Distributed agreement in the presence of communication and process failures. In Proceedings of the 14th IEEE Convention of Electrical \& Electronics Engineers in Israel. IEEE, March 1985.

[26] O. Rutti, Z. Milosevic, and A. Schiper. Generic construction of consensus algorithms for benign and byzantine faults. Dependable Systems and Networks, International Conference on, 0:343-352, 2010.

[27] N. Santoro and P. Widmayer. Time is not a healer. In Proc. 6th Annual Symposium on Theor. Aspects of Computer Science (STACS'89), volume 349 of LNCS, pages 304-313, Paderborn, Germany, Feb. 1989. Springer-Verlag.
[28] N. Santoro and P. Widmayer. Agreement in synchronous networks with ubiquitous faults. Theor. Comput. Sci., 384(2-3):232-249, Oct. 2007.

[29] H. M. Sayeed, M. Abu-Amara, and H. Abu-Amara. Optimal asynchronous agreement and leader election algorithm for complete networks with byzantine faulty links. Distrib. Comput., 9:147-156, December 1995.

[30] U. Schmid, B. Weiss, and I. Keidar. Impossibility results and lower bounds for consensus under link failures. SIAM J. Comput., 38(5):1912-1951, 2009.

[31] U. Schmid, B. Weiss, and J. Rushby. Formally verified byzantine agreement in presence of link faults. In 22nd International Conference on Distributed Computing Systems (ICDCS'02), pages 608-616, Vienna, Austria, July 2-5, 2002.

[32] U. Schmid, B. Weiss, and J. Rushby. Formally verified byzantine agreement in presence of link faults. In Proceedings of the 22 nd International Conference on Distributed Computing Systems (ICDCS'02), ICDCS '02, pages 608-, Washington, DC, USA, 2002. IEEE Computer Society.

[33] H.-S. Siu, Y.-H. Chin, and W.-P. Yang. Byzantine agreement in the presence of mixed faults on processors and links. IEEE Trans. Parallel Distrib. Syst., 9:335-345, April 1998. 